ESAIM: PROCEEDINGS, April 2009, Vol. 26, p. 65-93

H. Ammari, Editor

\title{
ON THE CONCEPT OF SCATTERED GAMMA-RAY IMAGING AND CORRESPONDING GENERALIZED RADON TRANSFORMS
}

\author{
M.K. NGUYEN ${ }^{1}$ And T. T. TRUONG ${ }^{2}$
}

\begin{abstract}
This paper reviews the concept of gamma-ray imaging based on Compton scattered radiation introduced some years ago. We describe its mathematical supporting structure of generalized Radon transforms and point out its main advantages in view of applications in nuclear medicine, industrial non-destructive evaluation, nuclear waste surveillance and fissile materials transportation monitoring. Some possible generalizations are mentioned as future research topics.
\end{abstract}

Résumé. Dans cet article, nous présentons le concept d'imagerie gamma basée sur le rayonnement diffusé par effet Compton, qui a été introduit quelques années auparavant. Nous exposons la structure mathématique des transformations de Radon généralisées sous-jacente et passons en revue ses propriétés avantageuses en vue d' applications en médecine nucléaire, contrôle non-destructif industriel, surveillance des dépôts de déchets radioactifs et contrôle du transport des matériaux fissiles. Quelques généralisations de ce concept sont susceptibles de recherche future.

\section{INTRODUCTION}

Gamma-ray imaging has emerged as an essential tool in areas such as high-energy astrophysics, national security and industrial non-destructive quality assessments [2], biomedical research and nuclear medicine [4], [10], where the availability functional information on human organs (tumors, brain, heart, etc.) or on metabolism helps to provide accurate diagnostic and efficient therapy in oncology, neurology, cardiology, etc. Generally the quality of gamma images is very poor as far as spatial resolution and signal-to-noise ratio are concerned. Gamma detectors are plagued by many degrading factors such as low performance of gamma optics, Poisson noise of photon emission, and several physical processes (attenuation, photoelectric absorption and scattering). In particular in low energy ranges, Compton scattering is dominant and causes deflection of photons as well as their energy loss. This leads to blurs, contrast reduction and consequently to poor image quality. A number of efforts are concentrated on the elimination of scattered photons. The usual remedy is to separate the scattered components from the non-scattered (primary) ones and only keep the primary events. Whatever the method

\footnotetext{
1 Laboratoire Equipes de Traitement des Images et du Signal, (CNRS UMR 8051/ENSEA/Université de Cergy-Pontoise),

6, avenue du Ponceau, 95014 Cergy-Pontoise, France.

2 Laboratoire de Physique Théorique et Modélisation (CNRS UMR 8089),

Université de Cergy-Pontoise, 95302 Cergy-Pontoise, France.
} 
used, scattered photons are either subtracted off from the overall counts or filtered by narrow energy windows. But it is not clear that discarding systematically Compton scattered photons is the best solution to be adopted.

In the present work, we propose an alternative approach to this problem by taking advantage of the properties of scattered photons, instead of discarding them as usually done. Actually we show that, not only these photons can be used to improve the image quality, as found at first [12], but also they generate an original new imaging process. In fact, we establish a new relation between the object radioactivity density and a series of images formed by the single-scattered photons and parameterized by various angles of scattering. This relation appears as a weighted sum of conical integral projections. It may be viewed as a Compound Conical Radon Transform integral of functions on cone surfaces [13]. Let us recall that the usual Radon transform (integrals of functions on lines or planes) is the corner stone of modern $X$-ray Computer Assisted Tomography (CAT). It turns out that the kernel of the new transformation, called Scatter Point Spread Function (SPSF), can be analytically computed. Moreover and most importantly, we prove that this new transformation is invertible and the kernel of the inverse transformation can be explicitly evaluated. These theoretical results allow the reconstruction of a 3D-object from a series of images parameterized by the angle of scattering (instead by an angle of spatial rotation). Consequently in this new imaging procedure, the recording of these images can be made without the motion of the detector as in the case of conventional tomography.

The idea of exploiting scattered radiation for imaging purposes has been proposed before but in other contexts [9] (e.g. Compton scattering Tomography [17] or electronic collimation in Compton camera [20], [3], [5], [8]). In Compton scattering Tomography, the aim is to determine the electron density in bulk matter (instead of the radioactivity density) from Compton scattered radiation from an $\gamma$-ray external pencil beam whereas, in emission imaging with a Compton camera, the efficiency of the detector is meant to be enhanced by the collecting of all Compton scattered photons through a planar scattering medium placed ahead of the detector. Thus the use of scattered radiation in these cases is very different from what we propose in this paper. Unfortunately no theoretical inversion formulas in three dimensions have been yet established for these cases.

This paper is organized as follows. We describe in chapter 2 the use of scattered radiation in emission imaging. By performing a physical analysis on single Compton-scattered photons we establish the so-called imaging equation which relates the single scattered photon flux density recorded on the detector to the radioactive volume density of the object and expressed as a Compounded Conical Radon Transform, hereafter coined as CCRT, see sections 2.1 to 2.3. We then give, in the next section, the explicit evaluation of the Point Spread Function, which should be named Scatter Point Spread Function or SPSF, in section 2.4. Next, we prove the most important property of this transform, which is the existence of an inverse mapping with an explicit computable kernel, see sections 2.6 and 2.7. The approach adopted here is different from the one adopted in our previous work [13] (there, an alternative treatment is given using the derivative of the imaging equation, which is free from formal divergences). Instead a direct treatment of this equation is presented and it leads to the expressions of the kernels of the Compound Conical Radon Transform and its inverse, from which an inversion theorem can be explicitly proved [14], [15]. To validate our approach, we present numerical calculations for actual image reconstruction, in section 2.8. Chapter 3 of the work is devoted to transmission imaging to design a new scheme for obtaining an accurate estimate of the attenuation map of the object and then propose a new scanning process in medical imaging. This process can be viewed as stationary transmission scanning using scattered radiation, see sections 3.1 - 3.2. An analysis of the algorithmic steps leading to the determination of the attenuation map will be given (section 3.3) as well as illustrations on medical phantoms (section 3.4). Finally, in chapter 4, we present an extension of this scattered radiation imaging principle to nondestructive evaluation in material sciences and preliminary results as illustrations. Conclusions and perspectives are given in the last section of the article. 


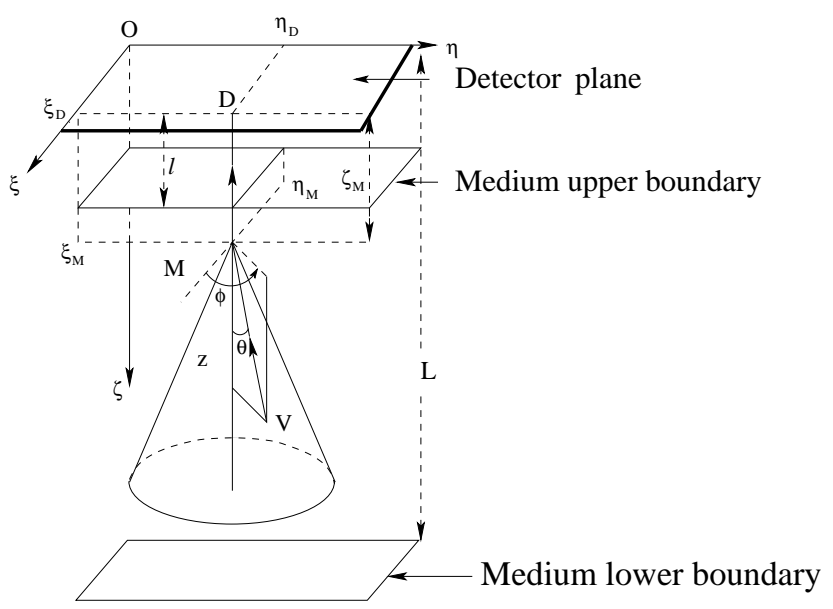

Figure 1. Coordinate system for the calculation of $\mathcal{T}$

\section{Scattered Radiation Single Photon Emission Imaging}

\subsection{Compton scattering}

Our point of view in this work is to focus on the emitted photons which undergo one Compton scattering and study how they may turn out to be relevant for image formation process [12].

First, we recall the relation between energy and scattering angle for the Compton-scattered photon [1]:

$$
E=E_{0} \frac{1}{1+\varepsilon(1-\cos \theta)}
$$

where $\theta$ is the scattering angle as measured from the incident photon direction; $E_{0}$, the photon initial energy; $\varepsilon=\frac{E_{0}}{m c^{2}}$ and $m c^{2}$ the rest energy of the electron . Eq. (1) shows that single-scattered photons have a continuous energy spectrum: $0 \leq E \leq E_{0}$, which is related to the scattering angle $\theta$. But Compton scattering is also a quantum phenomena: the emergence of the scattered photon has a probability of occurrence given by the Compton differential cross-section :

$$
\frac{d \sigma}{d \Omega}=\frac{r_{e}^{2}}{2} P(\theta)
$$

where $r_{e}$ is the classical radius of the electron and $P(\theta)$ the so-called Klein-Nishina probability for deflection by an angle $\theta$ :

$$
P(\theta)=\frac{1}{[1+\varepsilon(1-\cos \theta)]^{2}}\left[1+\cos ^{2} \theta+\frac{\varepsilon^{2}(1-\cos \theta)^{2}}{1+\varepsilon(1-\cos \theta)}\right] .
$$

As a result of Compton scattering, photons leaving an emitting point source can enter the detector along the collimator's direction after one or more scattering events. However, since single-scattered photons dominate the process [23], we shall limit ourselves in this paper to single-scattered photons.

\subsection{Image formation in scattered ray emission imaging with a gamma camera}

Thus at a given angle $\theta$, let $\tilde{g}(\mathbf{D}, \theta)$ be the photon flux density at detector site $\mathbf{D}$. This quantity describes essentially a secondary emission imaging process since it is based on secondary emission sites formed by the free electrons of the surrounding medium. In conventional image processing for $\gamma$-ray camera, they are discarded by filtering or by other correction techniques. We study now the secondary image formation process by such scattered photons. 
Let $\mathbf{V}$ denote an point source object of coordinates $\left(\xi_{V}, \eta_{V}, \zeta_{V}\right)$ and $f(\mathbf{V})$ be the object density function, defined as $f(\mathbf{V})=f\left(\xi_{V}, \eta_{V}, \zeta_{V}\right)$. It represents the number of photons emitted per unit time and per unit object (or source) volume, uniformly distributed around the $4 \pi$ solid angle at site $\mathbf{V}$. $f(\mathbf{V})$ is a positive function with compact support in $\mathbb{R}^{3}$. Thus in one given direction, making an angle $\theta$ with the vertical downward direction $O \zeta$ (see Figure 1), the number of photons emitted in a small solid angle $d \Omega_{M}$ around site $\mathbf{M}$ by an elemental source volume $d \mathbf{V}$, per unit time is:

$$
\frac{1}{4 \pi} f(\mathbf{V}) d \mathbf{V} d \Omega_{M}
$$

Now, by definition of the solid angle we have $d \Omega_{M}=\frac{d \sigma_{M}}{M V^{2}}$, where $d \sigma_{M}$ is the area element around site $\mathbf{M}$ normal to the direction $V M$. Consequently the flux of photons arriving at $\mathbf{M}$ in the $V M$ direction is:

$$
\frac{f(\mathbf{V}) d \mathbf{V}}{4 \pi} \frac{1}{M V^{2}} \exp (-\mu r)
$$

where $r=V M$ and $\mu$ is the linear mean coefficient of absorption in biological tissues (i.e. organ and/or surrounding medium).

But site $\mathbf{M}$ is located either inside the studied organ or inside the surrounding biological medium, and there are $n_{e}(\mathbf{M}) d \mathbf{M}$ free electrons in a small volume element $d \mathbf{M}$. Note that in general the density of electrons is not uniform. Now since $\left(\frac{d \sigma}{d \Omega}\right) d \Omega_{D}$ is the differential cross-section of photons scattering in the $\theta$-direction, and since $d \Omega_{D}=\frac{d \sigma_{D}}{M D^{2}}$, the number of photons reaching a unit detector surface at $\mathbf{D}$ per unit time (after division by the area $\left.d \sigma_{D}\right)$ is the flux density recorded by the detector at site $\mathbf{D}$ :

$$
\frac{f(\mathbf{V}) d \mathbf{V}}{4 \pi} \frac{e^{-\mu r}}{M V^{2}} n_{e}(\mathbf{M}) d \mathbf{M} \frac{r_{e}^{2}}{2} P(\theta) \frac{e^{-\mu\left(\zeta_{M}-l\right)}}{M D^{2}},
$$

which depends on $d \mathbf{V}$ and $d \mathbf{M}$.

Consequently, the number of photons recorded per unit time and unit detector area at site $\mathbf{D}=\left(\xi_{D}, \eta_{D}\right)$, $\tilde{g}(\mathbf{D}, \theta)$, is due to all emitting point sources $\mathbf{V}$ situated on a cone with opening angle $\theta$, the axis parallel to $O \zeta$ and with apex at the scattering site on the vertical line $M D$ :

$$
\begin{gathered}
\tilde{g}(\mathbf{D}, \theta)=\int d \xi_{M} d \eta_{M} \frac{d \zeta_{M}}{\zeta_{M}^{2}} \delta\left(\xi_{D}-\xi_{M}\right) \delta\left(\eta_{D}-\eta_{M}\right) n_{e}(\mathbf{M}) e^{-\mu\left(\zeta_{M}-l\right)} \\
\int \frac{f(\mathbf{V}) d \mathbf{V}}{4 \pi} \frac{\delta(\text { Cone }) \exp (-\mu r)}{M V^{2}} \frac{r_{e}^{2}}{2} P(\theta),
\end{gathered}
$$

where $\delta$ (Cone) restricts the integration over $\mathbf{V}$ to the circular cone. In fact the cone integral has appeared first in the process of image generation in a class Compton cameras [5]. If one uses the local spherical coordinates centered at $\mathbf{M}$ in Figure 1, we have $d \mathbf{V}=r^{2} d r \sin \alpha d \alpha d \phi$ then $\delta($ Cone $)=\frac{1}{r} \delta(\theta-\alpha)$. Clearly $\tilde{g}(\mathbf{D}, \theta)$ has the dimension of a photon flux through a plane (flux density) and is a positive function with compact support on $\mathbb{R}^{2}$.

Note that the function of three variables $f(\mathbf{V})$ is mapped onto $\tilde{g}(\mathbf{D}, \theta)$, which is also a function of three scalar variables, since $\mathbf{D} \in \mathbb{R}^{2}$ and $\theta \in \mathbb{R}$.

\subsection{The Compounded Conical Radon Transform or CCRT}

In order to focus only on Compton effect features, other image degradations (absorption, imperfect detector) are now overlooked and we set $n_{e}=$ constant and $\mu=0$ for simplicity. This is a plausible hypothesis since soft biological tissues have a mass density close to that of water [1].

In Figure 1, the measuring apparatus formed by the collimator, the detector and the photomultiplier bank is collapsed into a rectangle in the horizontal plane $O \xi \eta$. Moreover, we assume that the object under study lies below the plane $\zeta=l$, i.e. it does not touch the detector plane. 
The coordinates of $\mathbf{V}$ in this system are:

$$
\begin{aligned}
\xi_{V} & =\xi_{M}+r \sin \theta \cos \phi, \\
\eta_{V} & =\eta_{M}+r \sin \theta \sin \phi, \\
\zeta_{V} & =\zeta_{M}+r \cos \theta,
\end{aligned}
$$

where $\theta$ is the scattering angle and $\phi$ the azimuthal angle of $\mathbf{V}$ with respect to the cone axis $D M$. The integration measure on the cone is $r \sin \theta d \phi d r$. Eq.4 after integration over $\xi_{M}$ and $\eta_{M}$ becomes our basic imaging equation:

$$
\begin{gathered}
\tilde{g}(\mathbf{D}, \theta)=\tilde{K}(\theta) \int_{l}^{\infty} \frac{d \zeta_{M}}{\zeta_{M}^{2}} \int_{0}^{2 \pi} d \phi \int_{0_{+}}^{\infty} \frac{d r}{r} \\
f\left(\xi_{D}+r \sin \theta \cos \phi, \eta_{D}+r \sin \theta \sin \phi, \zeta_{M}+r \cos \theta\right),
\end{gathered}
$$

where the factor $\tilde{K}(\theta)$ contains terms dependent on $\theta$ :

$$
\tilde{K}(\theta)=\frac{n_{e}}{4 \pi} \frac{r_{e}^{2}}{2} P(\theta) \sin \theta
$$

In this expression of $\tilde{g}(\mathbf{D}, \theta)$, the integral on $r$ is formally divergent near the origin since $f(\mathbf{V})$ is usually a bounded function (the activity density is everywhere finite). Mathematically, the regularization of the integral in Eq. (6) is achieved by assigning a cut-off for the integration in $r$, here the lower integration bound is noted $0_{+}$. In fact in practical numerical calculations this cut-off can be easily accomplished by appropriate discretization of the integration range.

As $f(\mathbf{V})$ is of compact support, the $\zeta$ integration can be limited to the interval $l<\zeta<L$, which simplifies a great deal the numerical treatment in a real computation of the image. This $\zeta$ integral can be understood as the sum, along a vertical line, of integrals of $f(\mathbf{V})$ on cones of axis parallel to $O \zeta$, apex $\mathbf{M}$ and opening angle $\theta$. The image of $f(\mathbf{V})$ is thus represented by a set of conical integral projections instead of planar integral projections. In this sense one may speak of a Compounded Conical Radon Transform of $f(\mathbf{V})$, or (CCRT).

To single out the imaging mechanism, we rewrite the CCRT in the Fredholm form using $t=\tan \theta, \tilde{K}(\theta)=$ $K(t)$ and $\tilde{g}(\mathbf{D}, \theta)=g(\mathbf{D}, t)$, as :

$$
g(\mathbf{D}, t)=\int d \mathbf{V} p(\mathbf{D}, t \mid \mathbf{V}) f(\mathbf{V})
$$

where $p(\mathbf{D}, t \mid \mathbf{V})$ stands as transformation kernel

$$
\begin{gathered}
p(\mathbf{D}, t \mid \mathbf{V})=K(t) \int_{l}^{\infty} \frac{d \zeta_{M}}{\zeta_{M}^{2}} \int_{0}^{\infty} \frac{d r}{r} \int_{0}^{2 \pi} d \phi \\
\delta\left(\xi-\xi_{D}-r \sin \theta \cos \phi\right) \delta\left(\eta-\eta_{D}-r \sin \theta \sin \phi\right) \delta\left(\zeta-\zeta_{M}-r \cos \theta\right) .
\end{gathered}
$$

Physically this kernel is also called the Point Spread Function (PSF), or image of a point source at site $\mathbf{V}=$ $(\xi, \eta, \zeta)$. Nevertheless this image is due to single scattered photons rather than primary photons, and it is called Scatter PSF (or SPSF).

\subsection{The Scatter Point Spread Function - SPSF- and its properties}

We are now in a position to evaluate explicitly the SPSF. Using the Fourier representation of the $\delta$-function, and computing the integral in $\zeta_{M}$ as follows:

$$
\int_{l}^{\infty} \frac{d \zeta_{M}}{\zeta_{M}^{2}} \delta\left(\zeta-\zeta_{M}-r \cos \theta\right)=\frac{Y(\zeta-l-r \cos \theta)}{(\zeta-r \cos \theta)^{2}}
$$


with $Y(\zeta)$ as the Heaviside unit step function. We can now represent the kernel $p(\mathbf{D}, t \mid \mathbf{V})$ by its Fourier transform $\mathcal{P}(u, v \mid \zeta, t)$ :

$$
p(\mathbf{D}, t \mid \mathbf{V})=\iint d u d v \mathcal{P}(u, v \mid \zeta, t) \exp 2 i \pi\left[\left(\xi-\xi_{D}\right) u+\left(\eta-\eta_{D}\right) v\right],
$$

with:

$$
\mathcal{P}(u, v \mid \zeta, t)=2 \pi K(t) \int_{0_{+}}^{\infty} \frac{d r}{r} J_{0}\left(2 \pi r \sin \theta \sqrt{u^{2}+v^{2}}\right) \frac{Y(\zeta-l-r \cos \theta)}{(\zeta-r \cos \theta)^{2}} .
$$

This result show that $\mathcal{P}(u, v \mid \zeta, t)$ is a function of the sole variable $\sqrt{u^{2}+v^{2}}$. We can now derive the SPSF by Fourier transformation. But in view of the rotational symmetry in Fourier space, the Fourier transform is equivalent to a Hankel transform of order zero [6]. If we introduce the following polar coordinates $\left(\xi-\xi_{D}\right)=$ $\rho \cos \alpha,\left(\eta-\eta_{D}\right)=\rho \sin \alpha$ and $u=\sigma \cos \psi, v=\sigma \sin \psi$, then :

$$
p(\mathbf{D}, t \mid \mathbf{V})=2 \pi \int_{0}^{\infty} \sigma d \sigma J_{0}(2 \pi \rho \sigma) \mathcal{P}(\sigma \mid \zeta, t)
$$

Now observing that $z=r \cos \theta=\rho / t$ where $t=\tan \theta$ and rearranging the expression of $\mathcal{P}(u, v \mid \zeta, t)$ as Hankel transform of order zero of the function (or distribution):

$$
\frac{1}{z^{2}} \frac{Y(\zeta-z-l)}{(\zeta-z)^{2}}
$$

we have:

$$
p(\mathbf{D}, t \mid \mathbf{V})=2 \pi \int_{0}^{\infty} \sigma d \sigma J_{0}(2 \pi \rho \sigma) \frac{K(t)}{t^{2}} 2 \pi \int_{0}^{\infty} \rho d \rho J_{0}(2 \pi \rho \sigma) \frac{1}{z^{2}} \frac{Y(\zeta-z-l)}{(\zeta-z)^{2}} .
$$

Now Hankel's identity [11]:

$$
(2 \pi)^{2} \int \sigma d \sigma J_{0}\left(2 \pi \rho^{\prime} \sigma\right) \int \rho d \rho J_{0}(2 \pi \rho \sigma) f(\rho)=f\left(\rho^{\prime}\right),
$$

allows to conclude that for scattering angles $0<\theta<\pi / 2,(t>0)$, the SPSF has the expression:

$$
\begin{aligned}
p(\mathbf{D}, t \mid \mathbf{V}) & =p\left(\xi_{D}, \eta_{D}, t \mid \xi, \eta \zeta\right) \\
& =t^{2} K(\theta) \frac{Y(t(\zeta-l)-\rho)}{\rho^{2}(t \zeta-\rho)^{2}} .
\end{aligned}
$$

The SPSF diverges as $\rho^{-2} \rightarrow \infty$ near the origin at constant $\theta$, when the scattering site $\mathbf{M}$ coincides with the point source $\mathbf{V}$. It also diverges when the scattering site $\mathbf{M}$ comes to the detection site $\mathbf{D}$, or when $t \zeta=\rho$, but this will not occur because of the limit $\zeta>l$ (recall that the object is not touching the detector plane). This kernel has also translational invariance in the detector plane:

$$
p(\mathbf{D}, t \mid \mathbf{V})=p\left(\left(\xi-\xi_{D}\right),\left(\eta-\eta_{D}\right) \mid t, \zeta\right) .
$$

Now for scattering angles $\theta$ in $[\pi / 2, \pi[$ or $(t<0)$, the SPSF is:

$$
p(\mathbf{D}, t \mid \mathbf{V})=t^{2} K(\theta) \frac{1}{\rho^{2}(|t| \zeta+\rho)^{2}} .
$$


There is no need to insert the Heaviside function because the scattering point lies always lower than the point source. To sum up we have the following expression for the SPSF:

$$
p(\mathbf{D}, t \mid \mathbf{V})=Y(t) t^{2} K(\theta) \frac{Y(t(\zeta-l)-\rho)}{\rho^{2}(t \zeta-\rho)^{2}}+Y(-t) t^{2} K(\theta) \frac{1}{\rho^{2}(|t| \zeta+\rho)^{2}},
$$

valid in the whole range of $t$ values.

It is noted that the SPSF has the general shape of a "Mexican hat" for $0<\theta<\pi / 2$ and of a simple peak at the origin for other angles. Moreover, it has rotational symmetry around the projection of the point source on the detector (horizontal) plane (see Figure 2). It is far from the gaussian shape - a simplified form - considered in most of the actual methods of treatment of scattered radiation.

As examples illustrating our idea of taking advantage of Compton-scattered rays, we present in Figure 3 an original object (disc of radius 6 units) and in Figure 4 some of its scattered-ray images at various deflection angles $\left(\theta=36^{0}, 53^{0}\right.$, and $90^{\circ}$, which correspond to energy losses of $-5 \%,-10 \%$ and $-22 \%$ of the incident photon energy). these images are observed on a gamma camera of dimensions 50 units $\times 50$ units. It is clear that the scattered-ray images carry useful information on the object structure and deserve to be used in image reconstruction. Considering them as a pure noise and discarding them as usually done is certainly not the most appropriate solution for the improvement of image quality.

\subsection{Remarks}

At this stage a few remarks are in order. Eq. (8) represents the images formed by single scattered photons. A similar equation can also be established for doubly scattered photons but the corresponding kernel is no longer simple because it is expressed as multiple integrals on different geometric variables as well as on intermediate energies. However the doubly scattered events have a very small occurence probability which is grossly equal to the square of the Klein-Nishina probability of Eq. (3). More generally $n^{\text {th }}$-order scattering events carrying the $n^{\text {th }}$-power of the Klein-Nishina term have even smaller occurence probability. Monte-Carlo studies [23] and experimental measurements [7] show that the first scattering is a dominant process compared to higher order scattering ones. As an example, in biological medium, single scattered radiation represents $36 \%$ of the total radiation against $4 \%$ of higher order scattered radiations. Thus the higher order scattering contributions with decreasingly low intensities, can be reasonably considered as noise with respect to the dominant contributions of single scattered events. In this context, the image formation by scattered radiation considered here can be regarded as to be unique with respect to the continuous form (infinite dimension) of the integral equation (see Eq. (8)). Of course there is another problem of uniqueness related to the discretized form (finite dimension) of the integral transform, but this is an entirely different problem.

A detailed study of higher order scattering effects would give an estimate on the accuracy of the present theory based on first order scattering events (some of our preliminary results on double scattering are presented in $[22])$. 

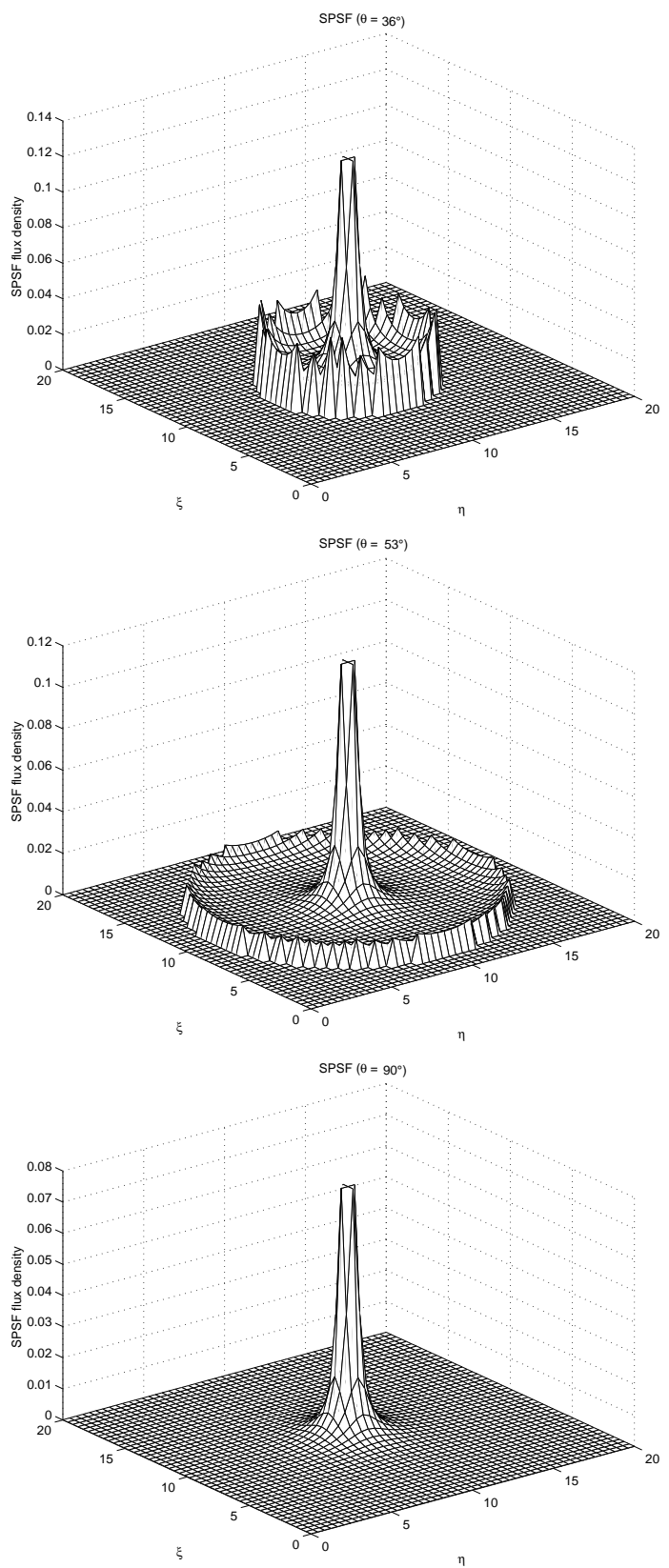

FigURE 2. SPSF of the transform $\mathcal{T}$ at various scattering angles $\left(\theta=36^{0}, 53^{0}\right.$ and $90^{\circ}$ respectively).

\subsection{Inversion of the Compounded Conical Radon Transform: the inverse kernel}

Having explicitly computed the kernel of the transformation $\mathcal{T}$, we show now that this transformation is invertible [13] and compute also the expression of the inverse kernel. Introducing the two-dimensional Fourier 

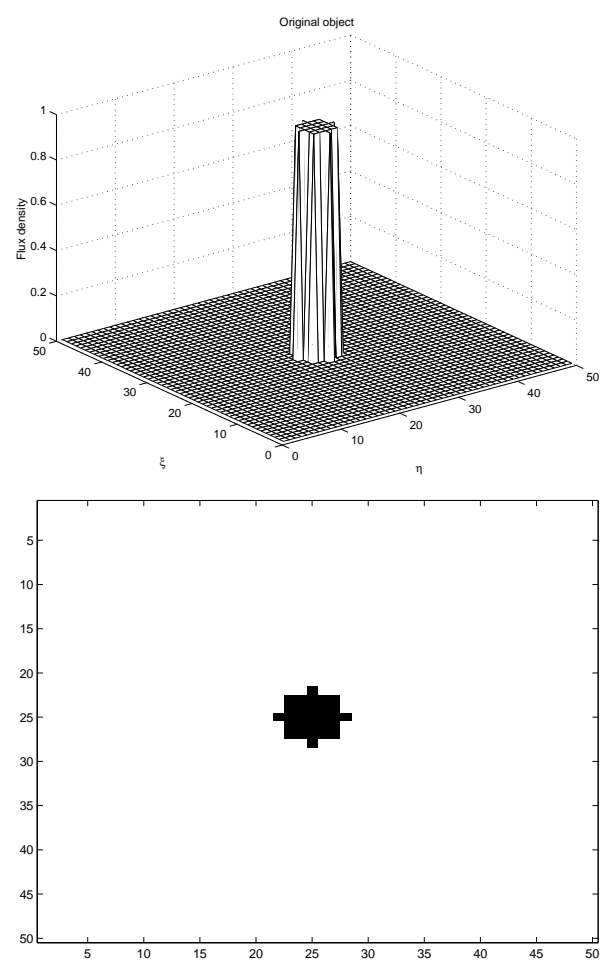

Figure 3. Original object (disc) and its cross-section.

transform $\tilde{F}\left(u, v, \zeta_{M} ; \theta\right)$ of $f(\mathbf{V})$ under the form:

$$
\tilde{F}\left(u, v, \zeta_{M} ; \theta\right)=\iint d \xi_{D} d \eta_{D} f\left(\xi_{D}, \eta_{D}, \zeta_{M} ; \theta\right) \exp -2 i \pi\left(u \xi_{D}+v \eta_{D}\right),
$$

and similarly $\tilde{G}(u, v ; \theta)$ for $\tilde{g}(\mathbf{D}, \theta)$, the imaging equation 6 reads now:

$$
\tilde{G}(u, v ; \theta)=2 \pi \tilde{K}(\theta) \int_{l}^{\infty} \frac{d \zeta_{M}}{\zeta_{M}^{2}} \int_{0_{+}}^{\infty} \frac{d r}{r} \tilde{F}\left(u, v, \zeta_{M}+r \cos \theta\right) J_{0}\left(2 \pi r \sin \theta \sqrt{u^{2}+v^{2}}\right) .
$$

Now using the variables $t$ and $z$ defined earlier, we can cast this last equation as a Hankel transform of order zero [6] (of course with the usual care for the lower bound of the integration):

$$
G(u, v ; t)=2 \pi K(t) \int_{0_{+}}^{\infty} z d z J_{0}\left(2 \pi z t \sqrt{u^{2}+v^{2}}\right) \frac{1}{z^{2}} \int_{l}^{\infty} \frac{d \zeta_{M}}{\zeta_{M}^{2}} F\left(u, v, \zeta_{M}+z\right) .
$$

The fact we integrate $z$ over $] 0, \infty[$, means that we consider here only scattering angles $\theta \in] 0, \pi / 2]$ or $t>0$ and $z>0$.

Thus inverting "formally" the Hankel transform (the lower integration bound is considered as to be 0), one obtains:

$$
\frac{1}{z^{2}} \int_{l}^{\infty} \frac{d \zeta_{M}}{\zeta_{M}^{2}} F\left(u, v, \zeta_{M}+z\right)=2 \pi\left(u^{2}+v^{2}\right) \int_{0_{+}}^{\infty} t d t J_{0}\left(2 \pi z t \sqrt{u^{2}+v^{2}}\right) \frac{G(u, v ; t)}{K(t)} .
$$



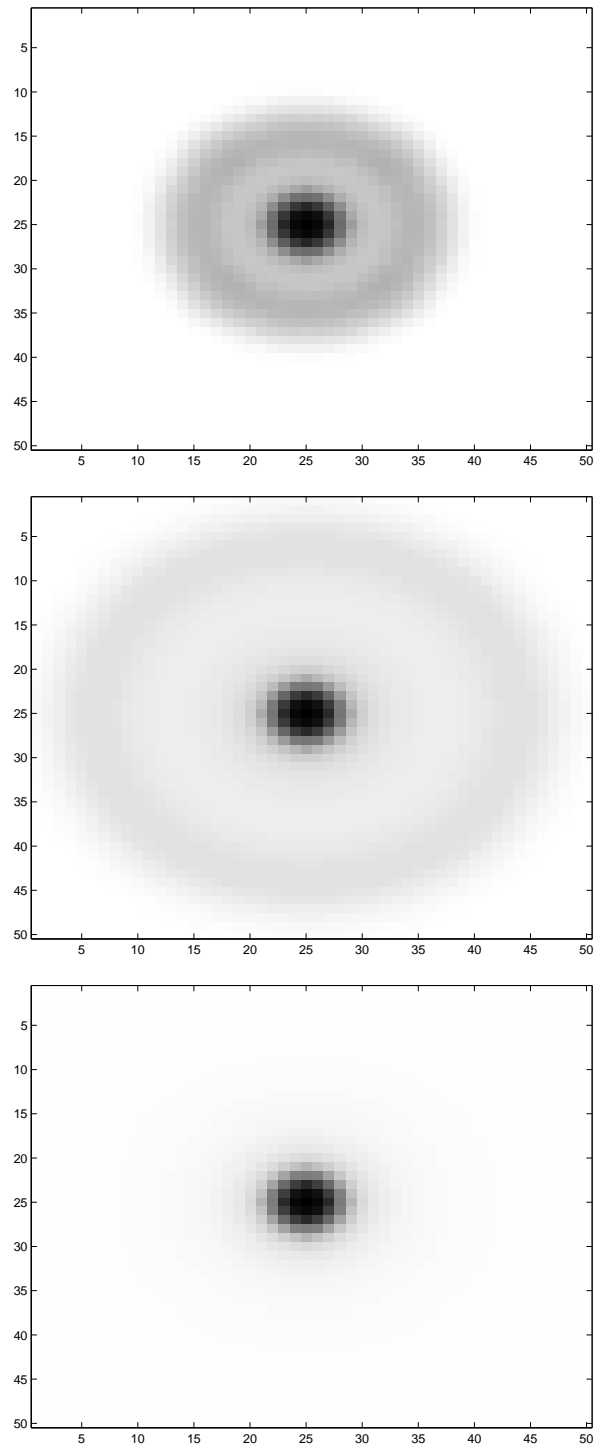

FiguRE 4. Series of secondary images parameterized by scattering angles $\left(\theta=36^{0}, 53^{0}\right.$ and $90^{0}$ respectively).

In the other range of $\theta$, i.e. $\theta \in[\pi / 2, \pi[$ we have $t<0$ and $z<0$, the previous relation becomes:

$$
\frac{1}{z^{\prime 2}} \int_{l}^{\infty} \frac{d \zeta_{M}}{\zeta_{M}^{2}} F\left(u, v, \zeta_{M}-z^{\prime}\right)=2 \pi\left(u^{2}+v^{2}\right) \int_{0_{+}}^{\infty} t^{\prime} d t^{\prime} J_{0}\left(2 \pi z^{\prime} t^{\prime} \sqrt{u^{2}+v^{2}}\right) \frac{G\left(u, v ;-t^{\prime}\right)}{K\left(-t^{\prime}\right)}
$$

where $z=-z^{\prime}$ and $t=-t^{\prime}$ with $z^{\prime}>0$ and $t^{\prime}>0$. We can put the two inverse cases together in a single formula, valid for all $t$, as follows:

$$
\int_{l}^{\infty} \frac{d \zeta_{M}}{\zeta_{M}^{2}} F\left(u, v, \zeta_{M}+z\right)=2 \pi\left(u^{2}+v^{2}\right) z^{2}
$$




$$
\int_{0_{+}}^{\infty} t d t J_{0}\left(2 \pi|z| t \sqrt{u^{2}+v^{2}}\right)\left[Y(z) \frac{G(u, v ; t)}{K(t)}+Y(-z) \frac{G(u, v ;-t)}{K(-t)}\right] .
$$

Now the left hand side of the previous equation can be put under an alternative form using Fourier analysis:

$$
\int_{l}^{\infty} \frac{d \zeta_{M}}{\zeta_{M}^{2}} F\left(u, v, \zeta_{M}+z\right)=\int_{-\infty}^{\infty} d w \mathcal{J}_{l}(w) \bar{F}(u, v, w) \exp [-2 i \pi w(l+z)]
$$

where $\bar{F}(u, v, w)$ is the three dimensional Fourier transform of $f(\mathbf{V}), \mathcal{J}_{l}$ the Fourier transform of $Y(s)(s+l)^{-2}$ is given in Fourier tables as:

$$
\mathcal{J}_{l}(w)=2 i \pi w\left\{e^{2 i \pi l w}[C i(2 \pi l|w|)-i \epsilon(w) S i(2 \pi l|w|)]-\frac{i}{2 \pi w l}\right\}
$$

here the variable $s$ is related to $\zeta_{M}$ by $\zeta_{M}=(s+l)$ and $\epsilon(w)$ is the sign function of $w$. The variable $(z+l)$ may take now any value in $\mathcal{R}$.

To extract $\bar{F}(u, v, w)$, we make an inverse Fourier transform on the last form of the imaging Eq. (23):

$$
\begin{gathered}
\bar{F}(u, v, w)=\frac{1}{\mathcal{J}_{l}(w)} \int_{-\infty}^{\infty} d z e^{2 i \pi w(z+l)}\left(u^{2}+v^{2}\right) z^{2} \\
2 \pi \int_{0_{+}}^{\infty} t d t J_{0}\left(2 \pi|z| t \sqrt{u^{2}+v^{2}}\right)\left[Y(z) \frac{G(u, v ; t)}{K(t)}+Y(-z) \frac{G(u, v ;-t)}{K(-t)}\right] .
\end{gathered}
$$

Finally, performing the three dimensional Fourier transform on the previous Eq. (26) and reexpressing $G(u, v ; t)$ as inverse two dimensional Fourier transform of $g(\mathbf{D}, t)$, the inverse transform $\mathcal{T}^{-1}$ can be put as:

$$
f(\mathbf{V})=\iint_{-\infty}^{\infty} d \mathbf{D} \int_{0}^{\infty} d t p^{-1}(\mathbf{V} \mid \mathbf{D}, t) g(\mathbf{D}, t)
$$

with the inverse kernel:

$$
\begin{gathered}
p^{-1}(\mathbf{V} \mid \mathbf{D}, t)=\iint_{-\infty}^{\infty} d u d v e^{2 i \pi\left[u\left(\xi-\xi_{D}\right)+v\left(\eta-\eta_{D}\right)\right]}\left(u^{2}+v^{2}\right) \\
2 \pi \int_{-\infty}^{\infty} z d z J_{0}\left(2 \pi|z| t \sqrt{u^{2}+v^{2}}\right) z H_{l}(\zeta+z+l) t \mathcal{K}_{t},
\end{gathered}
$$

where:

and $\mathcal{K}_{t}$ is a discrete linear operator defined by:

$$
\int_{-\infty}^{\infty} d w \exp [2 i \pi(\zeta+z+l) w] \frac{1}{\mathcal{J}_{l}(w)}=H_{l}(\zeta+z+l)
$$

$$
\mathcal{K}_{t} g(\mathbf{D}, t)=\left[Y(z) \frac{g(\mathbf{D}, t)}{K(t)}+Y(-z) \frac{g(\mathbf{D},-t)}{K(-t)}\right]
$$

Now by splitting up the integration range of $z$ into two parts: $]-\infty, 0]$ and $[0, \infty[$ and taking care of the action of $\mathcal{K}_{t}$, we obtain a new form of the inversion formula:

$$
\begin{gathered}
f(\mathbf{V})=\int_{-\infty}^{\infty} d \xi_{D} \int_{-\infty}^{\infty} d \eta_{D} \int_{0}^{\infty} d t \iint_{-\infty}^{\infty} d u d v e^{2 i \pi\left[u\left(\xi-\xi_{D}\right)+v\left(\eta-\eta_{D}\right)\right]}\left(u^{2}+v^{2}\right) \\
2 \pi \int_{0}^{\infty} z d z J_{0}\left(2 \pi|z| t \sqrt{u^{2}+v^{2}}\right) z\left[H_{l}(\zeta+z+l) \frac{\operatorname{tg}(\mathbf{D}, t)}{K(t)}+H_{l}(\zeta-z+l) \frac{\operatorname{tg}(\mathbf{D},-t)}{K(-t)}\right] .
\end{gathered}
$$


The integration on $z$ in Eq. (29) may be viewed as a Hankel transform of the function $z H_{l}(\zeta \pm z+l)$ :

$$
2 \pi \int_{0}^{\infty} z d z J_{0}\left(2 \pi|z| t \sqrt{u^{2}+v^{2}}\right) z H_{l}(\zeta \pm z+l)=\mathcal{H}^{ \pm}\left(\zeta+l, t \sqrt{u^{2}+v^{2}}\right) .
$$

Then the two dimensional Fourier transform of the kernel $p^{-1}(\mathbf{V} \mid \mathbf{D}, t)$ reads simply:

$$
\mathcal{P}^{-1}(u, v \mid \zeta, t)=\left(u^{2}+v^{2}\right) \mathcal{K}_{t}\left(u^{2}+v^{2}, \zeta\right),
$$

where $\mathcal{K}_{t}\left(u^{2}+v^{2}, \zeta\right)$ is a linear discrete operator defined by:

$$
\mathcal{K}_{t}\left(u^{2}+v^{2}, \zeta\right) g(\mathbf{D}, t)=\left[\frac{t \mathcal{H}^{+}\left(\zeta+l, t \sqrt{u^{2}+v^{2}}\right)}{K(t)} g(\mathbf{D}, t)+\frac{t \mathcal{H}^{-}\left(\zeta+l, t \sqrt{u^{2}+v^{2}}\right)}{K(-t)} g(\mathbf{D},-t)\right] .
$$

The integration over $t$ in Eq. (29) has the meaning that one must use a series of images labelled by the scattering angle $\theta$ to reconstruct the object. Thus this angle $\theta$ plays the role of a scanning parameter (spatial rotation angle) in a standard tomographic imaging procedure. This inverse kernel has obviously translational invariance in the detector plane. Let $\mathbf{S}$ be the projection of $\mathbf{V}$ on the detector plane, then we may write:

$$
\begin{gathered}
p^{-1}(\mathbf{V} \mid \mathbf{D}, t)=p^{-1}(\mathbf{S}, \zeta \mid \mathbf{D}, t)=p^{-1}(\mathbf{D S} \mid \zeta, t) . \\
=\iint_{-\infty}^{\infty} d u d v e^{2 i \pi\left[u\left(\xi-\xi_{D}\right)+v\left(\eta-\eta_{D}\right)\right]}\left(u^{2}+v^{2}\right) \mathcal{K}_{t}\left(u^{2}+v^{2}, \zeta\right) .
\end{gathered}
$$

\subsection{Proof of the inversion relation}

In this section we shall give the proof of the relation:

$$
\int d \mathbf{D} d t p^{-1}(\mathbf{V} \mid \mathbf{D}, t) p^{*}\left(\mathbf{D}, t \mid \mathbf{V}^{\prime}\right)=\delta\left(\mathbf{V}-\mathbf{V}^{\prime}\right)
$$

where the star means complex conjugate. By going to the two dimensional Fourier transform, the left hand side can be written as:

$$
\int_{0}^{\infty} d t \iint_{-\infty}^{\infty} d u d v e^{2 i \pi\left[u\left(\xi-\xi^{\prime}\right)+v\left(\eta-\eta^{\prime}\right)\right]} \mathcal{P}^{-1}(u, v \mid \zeta, t) \mathcal{P}\left(u, v \mid \zeta^{\prime}, t\right)
$$

In order to perform this calculation an alternative form for $\mathcal{P}\left(u, v \mid \zeta^{\prime}, t\right)$ using a Fourier representation is needed. We first express:

$$
\frac{Y\left(\zeta^{\prime}-z^{\prime}-l\right)}{\left(\zeta^{\prime}-z^{\prime}\right)^{2}}=\int_{-\infty}^{\infty} d w^{\prime} \mathcal{J}_{l}\left(w^{\prime}\right) e^{2 i \pi\left(\zeta^{\prime}-z^{\prime}-l\right) w^{\prime}}
$$

and insert it in:

$$
\mathcal{P}(u, v \mid \zeta, t)=2 \pi K(t) \int_{0_{+}}^{\infty} \frac{d z^{\prime}}{z^{\prime}} J_{0}\left(2 \pi z^{\prime} t \sqrt{u^{2}+v^{2}}\right) \int_{-\infty}^{\infty} d w^{\prime} \mathcal{J}_{l}\left(w^{\prime}\right) e^{2 i \pi\left(\zeta^{\prime}-z^{\prime}-l\right) w^{\prime}} .
$$

Now using the expression of $\mathcal{P}^{-1}(u, v \mid \zeta, t)$ in Eq. ( 31$)$ and the previous form of $\mathcal{P}\left(u, v \mid \zeta^{\prime}, t\right)$ we can perform the $t$ integration in:

$$
\begin{gathered}
\int_{0}^{\infty} d t \mathcal{P}^{-1}(u, v \mid \zeta, t) \mathcal{P}\left(u, v \mid \zeta^{\prime}, t\right)= \\
2 \pi \int_{0}^{\infty} d t K(t) \int_{0_{+}}^{\infty} \frac{d z^{\prime}}{z^{\prime}} J_{0}\left(2 \pi z^{\prime} t \sqrt{u^{2}+v^{2}}\right)
\end{gathered}
$$




$$
\begin{gathered}
\int_{-\infty}^{\infty} d w^{\prime} \mathcal{J}_{l}\left(w^{\prime}\right) e^{2 i \pi\left(\zeta^{\prime}-z^{\prime}-l\right) w^{\prime}} 2 \pi \frac{t}{K(t)}\left(u^{2}+v^{2}\right) \int_{-\infty}^{\infty} z d z J_{0}\left(2 \pi|z| t \sqrt{u^{2}+v^{2}}\right) z \\
\int_{-\infty}^{\infty} d w \frac{e^{2 i \pi(\zeta+z+l) w}}{\mathcal{J}_{l}(w)}
\end{gathered}
$$

and we see here that the $K(t)$ factors cancel out. The integration on $t$ can be carried out using the Bessel identity:

$$
\int_{0}^{\infty} \sigma d \sigma J_{0}\left(z^{\prime} \sigma\right) J_{0}(z \sigma)=\frac{1}{z^{\prime}} \delta\left(z-z^{\prime}\right) .
$$

Then after integration over $d z$ for $t>0$ and also $z>0$, we get:

$$
\begin{gathered}
\int_{0}^{\infty} d t \mathcal{P}^{-1}(u, v \mid \zeta, t) \mathcal{P}\left(u, v \mid \zeta^{\prime}, t\right)=\int_{0}^{\infty} \frac{d z^{\prime}}{z^{\prime}} \int_{-\infty}^{\infty} d w^{\prime} \mathcal{J}_{l}\left(w^{\prime}\right) e^{2 i \pi\left(\zeta^{\prime}-z^{\prime}-l\right) w^{\prime}} \\
\frac{1}{z^{\prime}} \delta\left(z-z^{\prime}\right) \int_{-\infty}^{\infty} z^{2} d z \int_{-\infty}^{\infty} d w \frac{1}{\mathcal{J}_{l}(w)} e^{2 i \pi(\zeta+z+l) w}
\end{gathered}
$$

The integration with respect to $z^{\prime}$ removes all the $z$ factors, and we are left with:

$$
\begin{gathered}
\int_{0}^{\infty} d t \mathcal{P}^{-1}(u, v \mid \zeta, t) \mathcal{P}\left(u, v \mid \zeta^{\prime}, t\right)=\int_{-\infty}^{\infty} d z e^{2 i \pi\left(w-w^{\prime}\right)} \int_{-\infty}^{\infty} d w^{\prime} \mathcal{J}_{l}\left(w^{\prime}\right) e^{2 i \pi w^{\prime}\left(\zeta^{\prime}-l\right)} \\
\int_{-\infty}^{\infty} d w \frac{1}{\mathcal{J}_{l}(w)} e^{2 i \pi w(\zeta+l)}=\int_{-\infty}^{\infty} d w d w^{\prime} \mathcal{J}_{l}\left(w^{\prime}\right) \frac{\delta\left(w-w^{\prime}\right)}{\mathcal{J}_{l}(w)} e^{2 i \pi w^{\prime}\left(\zeta^{\prime}-l\right)} e^{2 i \pi w(\zeta+l)} \\
=\int_{-\infty}^{\infty} d w e^{2 i \pi\left(\zeta^{\prime}-\zeta\right) w}=\delta\left(\zeta^{\prime}-\zeta\right)
\end{gathered}
$$

The remaining integrations over $d u$ and $d v$ yield obviously $\delta\left(\xi^{\prime}-\xi\right) \delta\left(\eta^{\prime}-\eta\right)$. The product of the kernels $p^{-1}(\mathbf{V} \mid \mathbf{D}, t)$ with the SPSF kernel $p(\mathbf{D}, t \mid \mathbf{V})$ is thus the identity [14].

\subsection{Numerical results on object reconstruction}

As an illustration of the inversion procedure discussed in the previous section, we present some numerical simulations showing how image reconstruction is realized using the analytic form of the inverse transform. They have been carried out under the following working conditions:

- the used $\gamma$-detector is a SPECT (Single Photon Emission Computed Tomography) camera. It has discretized dimensions $N$ length units $\times N$ length units. We have chosen $N=16$ to keep the volume of calculations reasonable.

- the scattering medium is represented by a cube of dimensions $N \times N \times N$,

- the electron density in biological medium is $n_{e}=3.5 \times 10^{23}$ electrons $/ \mathrm{cm}^{3}$,

- the radio pharmaceutical employed is Technecium 99 with an activity density $2.2 \times 10^{-2} \mathrm{Ci} / \mathrm{cm}^{3}$,

- the acquisition time per image is $0.1 \mathrm{sec}$,

- the 3D original object (cylinder of height 6 units) is placed at the center of the scattering medium (cube),

- the distance from camera to the upper face of the scattering medium cube is $l=200$ units.

Figure $5 \mathrm{a}$ represents the original object. Figure $5 \mathrm{~b}$ shows the series of images of the object at various scattering angles $\theta\left(5^{0}<\theta<175^{0}\right)$. These images, respectively without and with Poisson emission noise, observed on the detector, are shown respectively in Figure $6 \mathrm{a}$ and Figure $6 \mathrm{~b}$.

In Figure $7 \mathrm{a}$, is the reconstructed object in the absence of noise with a RMSE $=1.2 \%$ whereas in Figure $7 \mathrm{~b}$ the reconstruction is realized in the presence of noise $(S / N=9.7 \mathrm{~dB})$ with a RMSE $=8.9 \%$, which is perfectly 

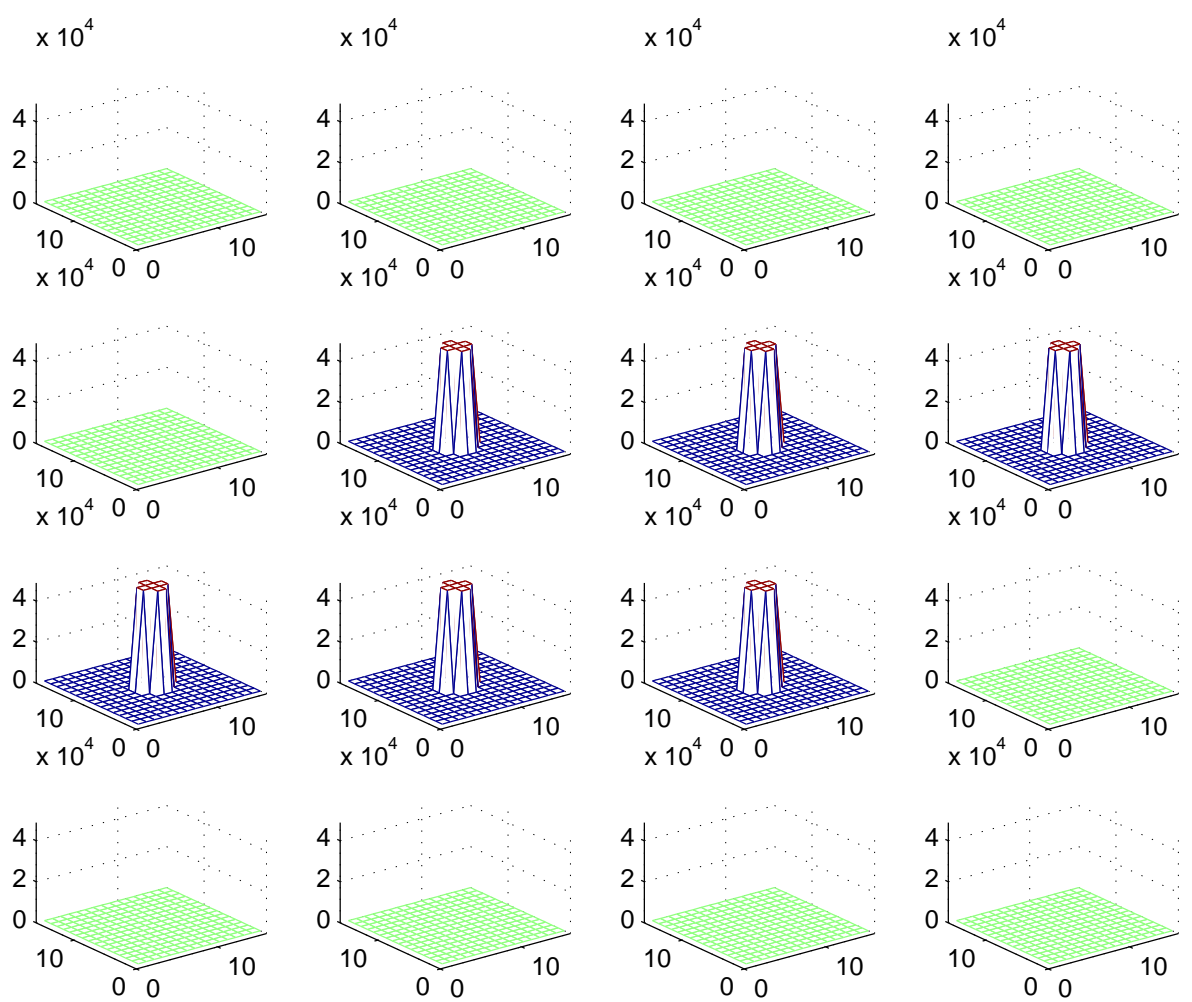

(a) Original object (cylinder) in a cube formed by 16 planes.
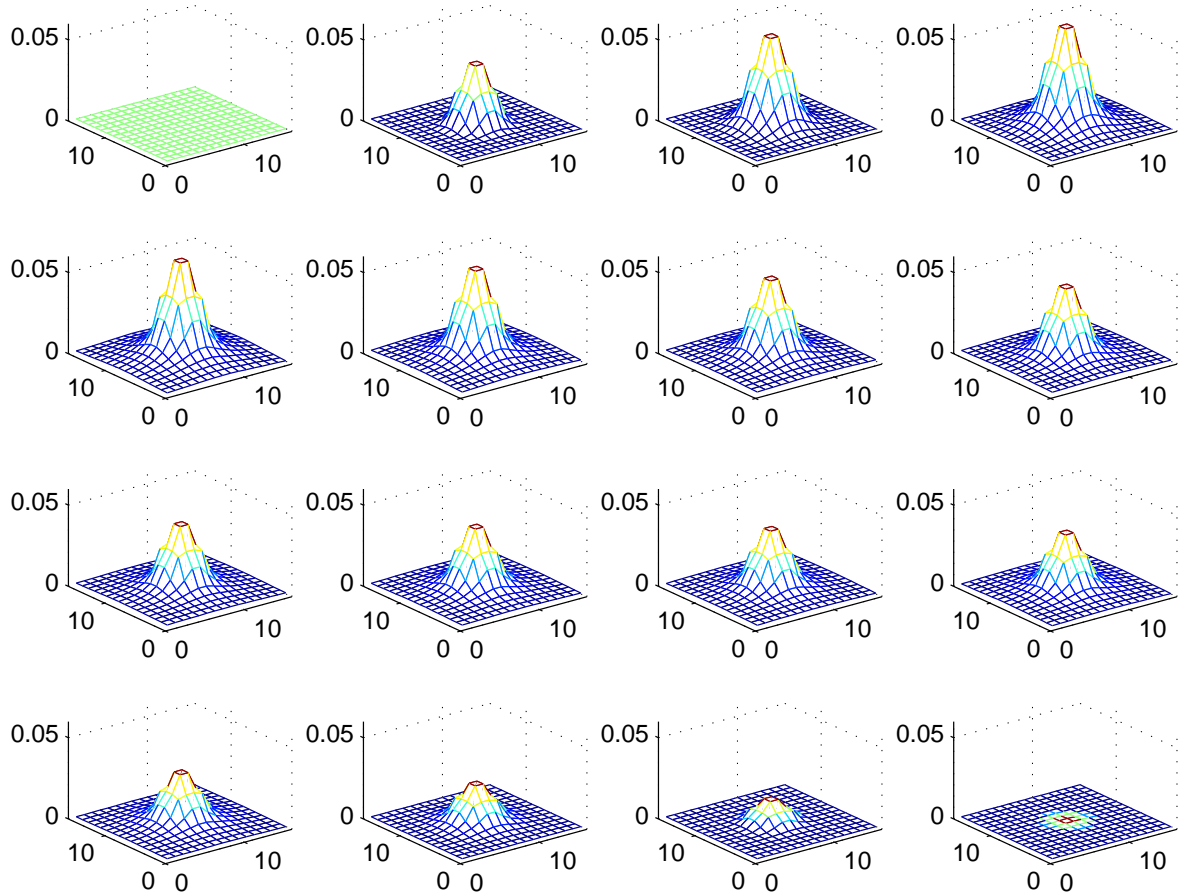

(b) Series of images parameterized by the angle of scattering $\theta\left(5^{0}<\theta<175^{0}\right)$.

Figure 5 

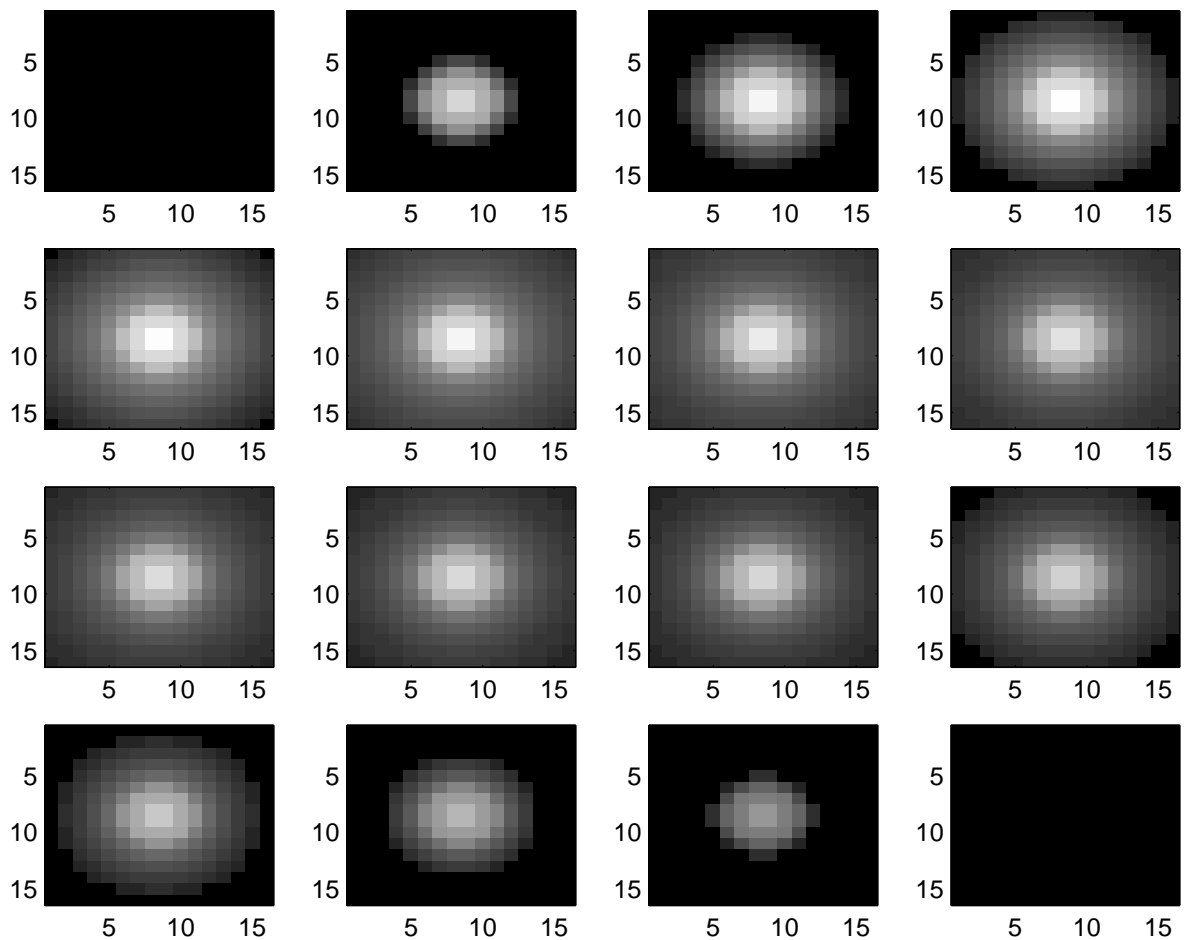

(a) Series of images without noise observed on the $\gamma$-camera.
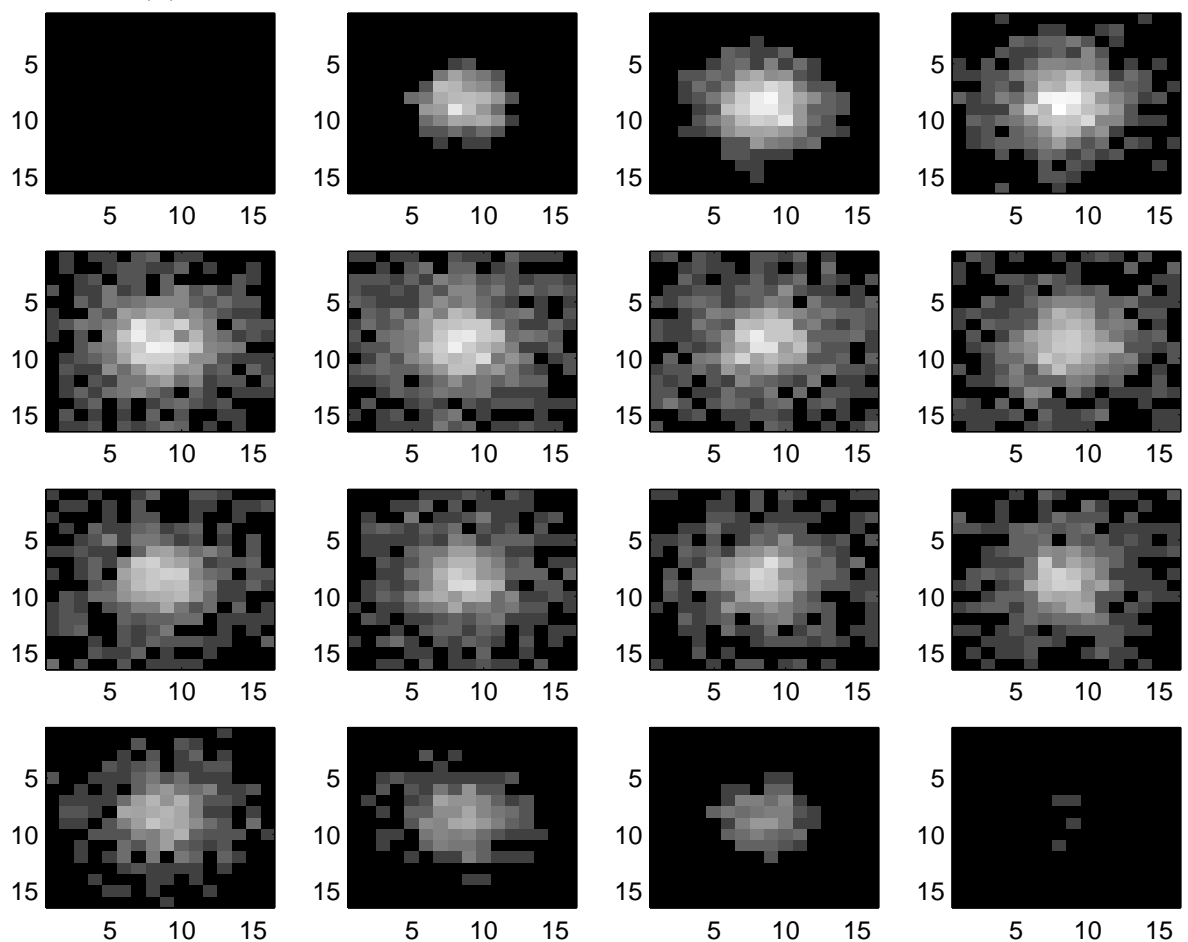

(b) Series of images with noise observed on the $\gamma$-camera $(S / N=9.7 \mathrm{~dB})$. 

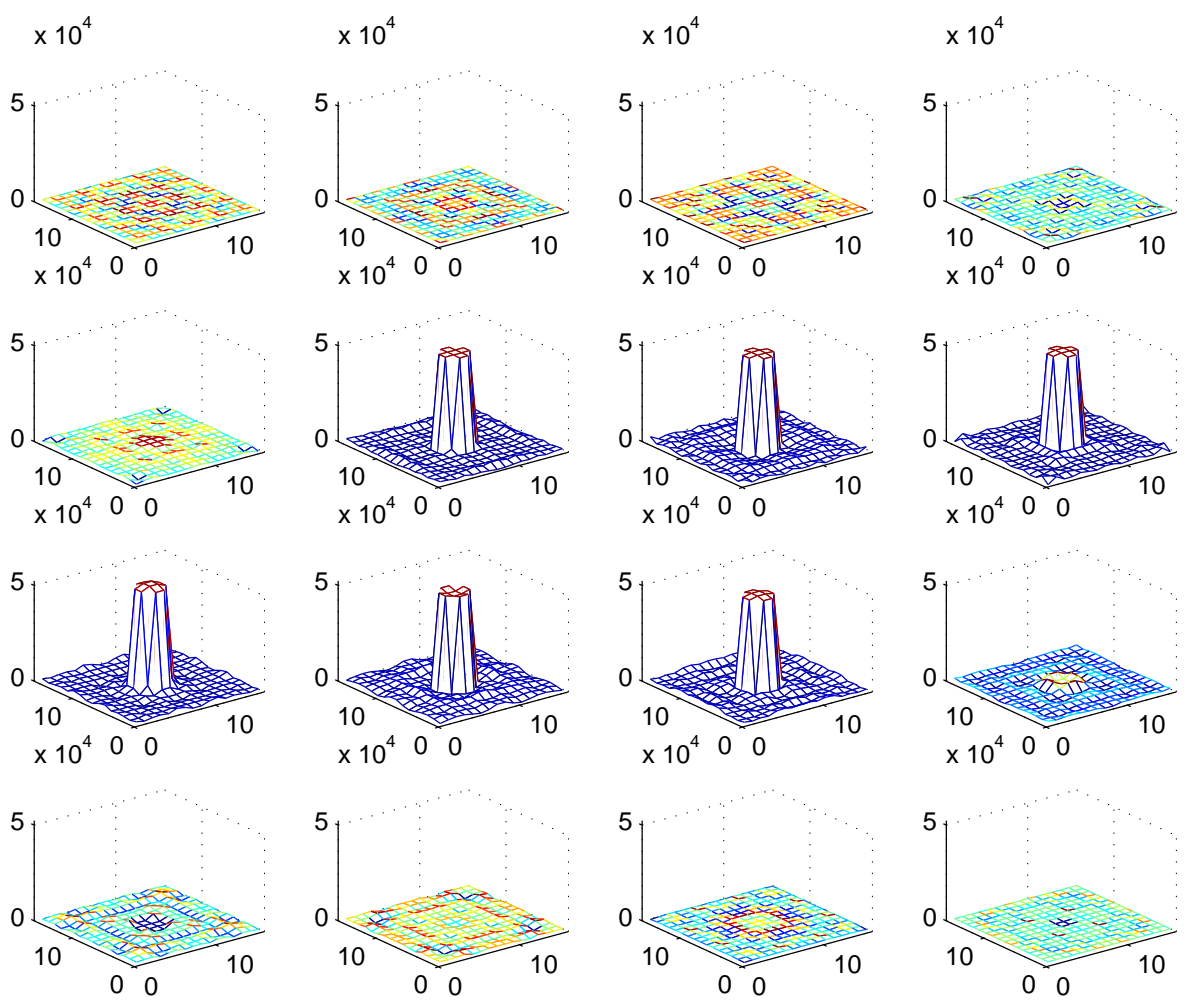

(a) Reconstructed object in the absence of noise (RMSE $=1.2 \%)$.

$\times 10^{4}$ $\times 10^{4}$ $\times 10^{4}$ $\times 10^{4}$
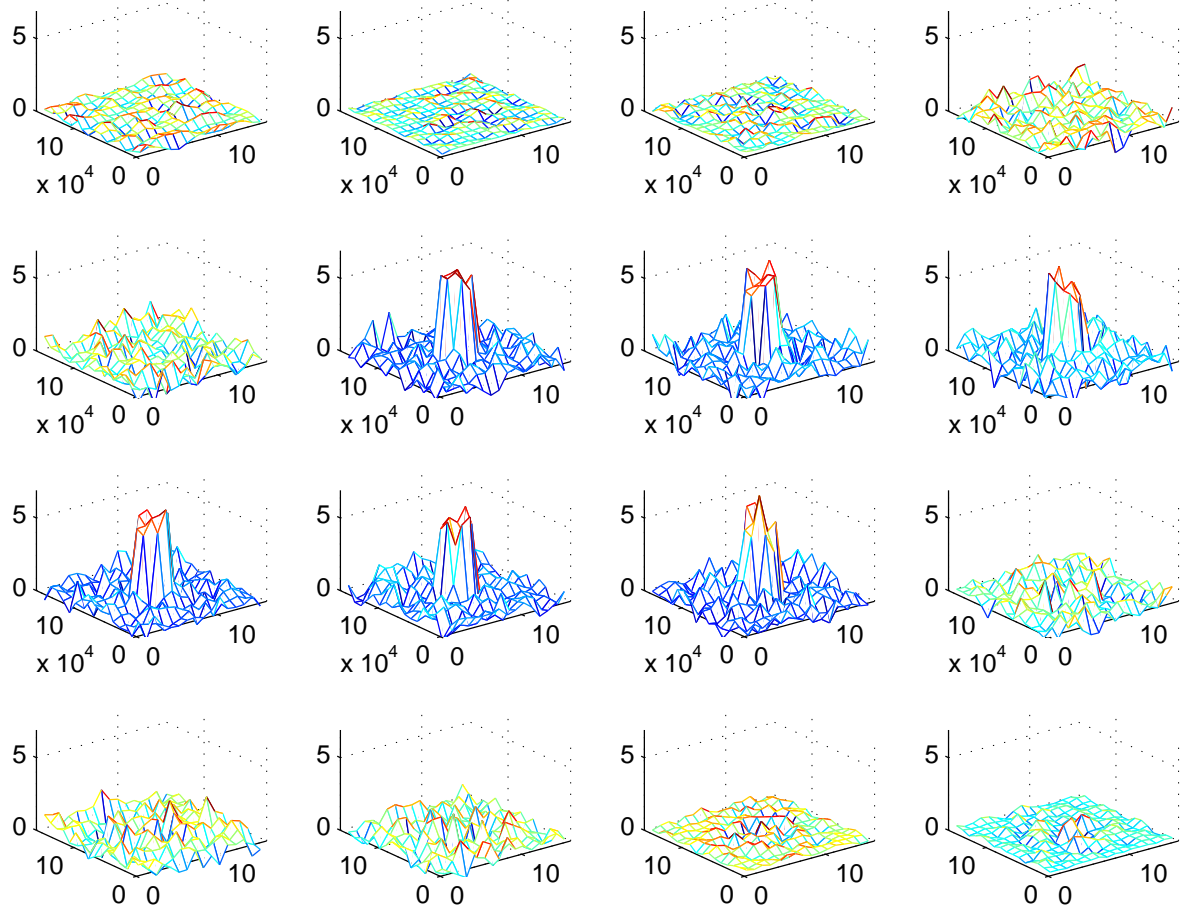

(b) Reconstructed object in the presence of noise (with $S / N=9.7 \mathrm{~dB}$ and RMSE $=8.9 \%$ ).

FIGURE 7 
reasonable. We observe a good performance of the Compound Conical Radon Transformation for modelling the new imaging process.

Concerning the resolution, the intrinsic resolution depends on the type of camera (collimator, crystal, photomultiplier and measurement electronics) used. Theoretical resolution is determined by the reconstruction method and its algorithm. The inclusion of scattered radiation increases considerably the number of detected photons, thus would contribute to improve the $\mathrm{S} / \mathrm{N}$ and the resolution of the imaging system. To evaluate accurately the resolution, it is necessary to have access to real data and to compare it with other methods which do not make use of scattered radiation. At the moment it is too early to use our preliminary results of simulation for this purpose. This work is ongoing with more realistic experimental conditions.

Since our main objective in this paper is to show how to exploit advantageously Compton scattered radiation to generate a new imaging principle we present only results on image formation as well as object reconstruction from scattered rays.

In real situations, of course, one must take into account other factors such as absorption by the medium, Poisson emission noise and the imperfections of the detector (collimator and measuring electronics).

The case of constant mean absorption (often assumed in the literature) is included in our recent work [14]. (The treatment of inhomogeneous absorption poses special mathematical difficulties. Recall that the inversion of the inhomogeneous attenuated X-ray transform in transmission imaging has been just found recently in 2001 [18], despite the fact that the inverse of the Radon transform is known since 1917).

Concerning emission noise there are well known methods to deal with it such as the method of Maximum Likelihood or the methods of wavelets. They may be used for "denoising" the measured data beforehand or jointly with the inversion process.

As for the imperfections of the detector, the standard way for treating this problem is to make use of a response function of the gaussian type operating with spatial coordinates as well as with energy coordinate. These issues are discussed in detail in [7].

\subsection{Applications}

In this new imaging principle, the fact that the usual spatial rotation angle is no longer needed for reconstructing an object, represents a major asset in instrumentation. The standard SPECT camera can still be used but now in a fixed position while imaging an object from a given view point. The reconstruction step is performed by computer and uses data collected by the camera at a series of values of the scattering angles.

This means that with such a detecting systems one can obtain the three dimensional radioactivity distribution of a radiating object (or of object which becomes radiating after treatment). In nuclear medicine, this is the case when the patient is injected with a radioactive tracer. In monitoring nuclear waste evolution, one can use this type of camera to have a three dimensional image of the activity distribution deep down in the dump. Finally a smaller model of this camera can be positioned discretely at key passage points to check illicit transport of fissile materials across nation borders.

\section{Scattered Radiation in Transmission Imaging: Determination of the Attenuation MaP}

\subsection{The Attenuation Problem in Gamma-ray Imaging}

In this section, we describe a novel method to determine the electron density of an object, which subsequently will lead to determination of its attenuation map.

In gamma ray imaging the attenuation problem is unavoidable and has to be met sooner or later. To gauge its importance let us go back one step and recall the image formation process. An object (e.g. human organ), 
in which a radioactive tracer (e.g. ${ }^{99 m} \mathrm{Tc}$ ) is injected, emits gamma photons of energy $E_{0}=140 \mathrm{keV}$. If the gamma camera is set to operate in stationary mode (from a fixed position) to record scattered photons (instead of primary photons) of lower energies $E(90 \mathrm{keV}<E<140 \mathrm{keV})$, then a series of images labeled by $E$ can be generated. If one considers that most of these photons will undergo single Compton scattering with electrons inside the object (since higher order scattering have much lower probability to be detected in this window, as reported in many Monte Carlo simulation studies [23], then the recorded data are directly related to the electronic distribution $n_{e}$. As shown in [13], the photon flux density measured on the gamma camera is expressed by the CCRT of the object activity density. Object reconstruction is consequently described by the inversion of the CCRT. This has been done in [14], at constant electronic density and without attenuating effects. However, photon scattering inside the medium will blur the recorded data and affect strongly the quality of the resulting reconstructions.

In general, to account correctly for attenuation effects, the image formation should be modeled by the attenuated Compounded Conical Radon Transform (or a-CCRT), in which attenuation factors are introduced along the photon paths. The a-CCRT formulation is similar to the well known attenuated X-ray transform. However, its inverse has not been found yet, even if the attenuation map is known before hand. In an ideal situation, one should be able to determine at the same time electron density (or attenuation map) and activity distribution from one set of measurements. Since this is not the case, we shall proceed by approximations as often done to tackle difficult problems. We begin by considering the CCRT formulation of the image formation process in the presence of nonuniform electron density $n_{e}$. This time we regard $n_{e}$ as unknown and assume the activity distribution to be given. In fact, the transmission scanning system simply consists of a calibrated point source positioned at a known site. The photon flux density measured by the camera gives a first direct estimate of $n_{e}$. We therefore propose an iterative method, based on the properties of the CCRT, to construct the object attenuation map. This method presents two main advantages:

- The attenuation map constructed in this way is photon energy dependent;

- The data acquisition procedure, which consists in collecting series of images at various scattering angles, is performed without having to move the detector.

As this new imaging concept uses scattered radiation, it requires high performance detector systems with excellent energy resolution. Thus, this concept is not meant to work on standard Anger cameras but might be applicable on new generation solid state systems such as cadmium zinc telluride (CZT) detector arrays. Possible angular uncertainties related to finite detector energy resolution may be teated as in the case of Compton cameras. It is recognized that ignoring higher order scattering owing to the complexity of the model is one major limitation of this work and will be targeted for further research. We describe first the working principles of our method and then expose the corresponding iterative attenuation correction algorithm.

\subsection{Electron density computation}

We establish now the relation giving the electron density in terms of the measured scattered photon flux density and the point source intensity. The reasoning goes along the lines exposed in previous sections for obtaining image formation.

Let $\mathbf{S}$ be the location of a point source of intensity $f_{0}$, which is the number of photons emitted uniformly per unit time around the $4 \pi$ solid angle (figure 8 ). The number of photons per unit time emitted along a direction $\mathbf{n}$ in a solid angle $d \Omega_{\mathbf{n}}$ is

$$
\frac{f_{0}}{4 \pi} d \Omega_{\mathbf{n}} .
$$

From this emitting site $\mathbf{S}$, there will be an incoming flux density on a scattering site $\mathbf{M}$ located at a distance $S M$ (with $S M=|\overrightarrow{S M}|$ ) from $\mathbf{S}$ equal to

$$
\frac{f_{0}}{4 \pi S M^{2}} \text {. }
$$


At this site $\mathbf{M}$, there are $n_{e}(\mathbf{M}) d \mathbf{M}$ electrons in a volume $d \mathbf{M}$ around $\mathbf{M}$. The number of scattered photons per unit area arriving at the detection site $\mathbf{D}$ on the gamma camera under a scattering angle $\theta$ is

$$
d g(\mathbf{D}, \theta)=\frac{f_{0}}{4 \pi S M^{2}} n_{e}(\mathbf{M}) d \mathbf{M} \frac{d \sigma^{C}}{d \Omega} \frac{1}{M D^{2}} \mathcal{A}_{\mu}(S M, M D)
$$

where $\frac{d \sigma^{C}}{d \Omega}$ is the Compton differential cross section given by the Klein-Nishina formula [1]. Since the product of the incoming flux density by the number of scatterers and by the Compton scattering cross section yields the number of particles scattered in the solid angle in the direction of angle $\theta$, we must introduce the factor $1 / M D^{2}$ (the square of the distance from site $\mathbf{M}$ to site $\mathbf{D}$ ), to get the flux density arriving at detection site $\mathbf{D}$.

$\mathcal{A}_{\mu}(S M, M D)$ represents the total attenuation factor from emission to detection

$$
\mathcal{A}_{\mu}(S M, M D)=\exp \left(-\int_{S M} \mu\left(\mathbf{r}, E_{0}\right) \delta(S M) d \mathbf{r}-\int_{M D} \mu(\mathbf{r}, E) \delta(M D) d \mathbf{r}\right),
$$

where $\mu(\mathbf{r}, E)$ is the linear attenuation coefficient at site $\mathbf{r}$ and energy $E$ which can be expressed in terms of $\sigma_{E}(\mathbf{r})$, the Compton cross section at energy $E$ of material at site $\mathbf{r}$, as:

$$
\mu(\mathbf{r}, E)=\sigma_{E}(\mathbf{r}) n_{e}(\mathbf{r})
$$

The delta distributions restrict the integration to lines $S M$ and $M D$ in equation (45).

The flux density at the detector per unit volume is a nonlinear functional of $n_{e}$ given by

$$
\frac{d g(\mathbf{D}, \theta)}{d \mathbf{M}}=\frac{f_{0}}{4 \pi} \frac{1}{S M^{2}} n_{e}(\mathbf{M}) \frac{d \sigma^{C}}{d \Omega} \frac{1}{M D^{2}} \mathcal{A}_{\mu}(S M, M D)
$$

Therefore obtaining $n_{e}$ directly from the measurement of $d g(\mathbf{D}, \theta) / d \mathbf{M}$ would not be possible. This is why an alternative strategy is proposed. The idea is to start first with a linearized form of equation (47), which is quite natural

$$
\frac{d g(\mathbf{D}, \theta)}{d \mathbf{M}}=\widetilde{g}(\mathbf{D}, \theta)=\frac{f_{0}}{4 \pi} \frac{1}{S M^{2}} n_{e}(\mathbf{M}) \frac{d \sigma^{C}}{d \Omega} \frac{1}{M D^{2}}
$$

which precisely amounts to neglecting the nonlinear factor $\mathcal{A}_{\mu}(S M, M D)$ and construct an efficient algorithm based on equation (48). This is done in the next subsection.

\subsection{Iterative Attenuation Correction algorithm (IAC) using scattered radiation}

Because of the presence of the collimator, site $\mathbf{M}$ is just situated on a line parallel to the collimator axis at site $\mathbf{D}$. So in an appropriately chosen coordinate system, we would have for a given point source located at $\mathbf{S}=\left(x_{S}, y_{S}, z_{S}\right),\left(x_{M}=x_{D}, y_{M}=y_{D}\right)$ and $\mathbf{D}=\left(x_{D}, y_{D}, 0\right)$. Consequently,

$$
\widetilde{g}(\mathbf{D}, \theta)=n_{e}\left(x_{M}, y_{M}, z_{M}\right) \frac{f_{0}}{4 \pi} \frac{d \sigma^{C}}{d \Omega} \frac{1}{\left(x_{M}-x_{S}\right)^{2}+\left(y_{M}-y_{S}\right)^{2}+\left(z_{M}-z_{S}\right)^{2}} \frac{1}{z_{M}^{2}},
$$

with

or alternatively

$$
z_{M}=z_{S}-\cot \theta \sqrt{\left(x_{M}-x_{S}\right)^{2}+\left(y_{M}-y_{S}\right)^{2}}
$$

$$
\begin{gathered}
\tilde{g}(\mathbf{D}, \theta)=n_{e}\left(x_{D}, y_{D}, z_{S}-\cot \theta \sqrt{\left(x_{D}-x_{S}\right)^{2}+\left(y_{M}-y_{S}\right)^{2}}\right) \\
\frac{f_{0}}{4 \pi} \frac{d \sigma^{C}}{d \Omega} \frac{\sin ^{2} \theta}{\left(x_{M}-x_{S}\right)^{2}+\left(y_{M}-y_{S}\right)^{2}} \frac{1}{\left(z_{S}-\cot \theta \sqrt{\left(x_{M}-x_{S}\right)^{2}+\left(y_{M}-y_{S}\right)^{2}}\right)^{2}} .
\end{gathered}
$$

Therefore one may rewrite equation (9) to extract the electronic density as 


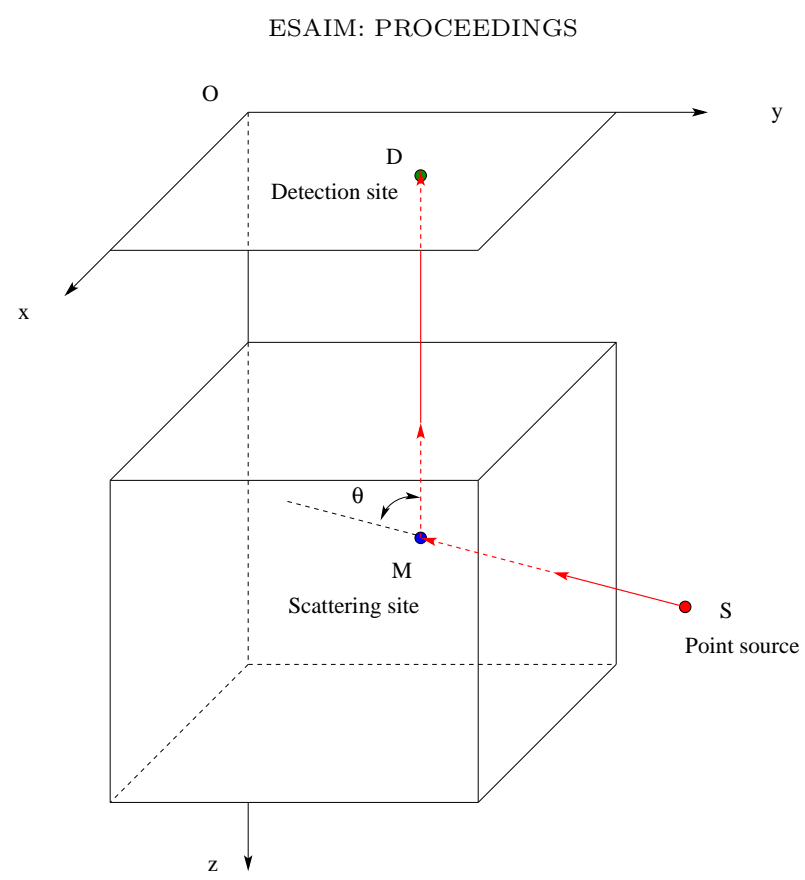

FIGURE 8. Geometric parameters of photon scattering in a medium resulting from point source transmission scanning.

$$
n_{e}\left(x_{M}, y_{M}, z_{M}\right)=\widetilde{g}\left(x_{M}, y_{M}, \theta\right) \frac{4 \pi}{f_{0}\left(\frac{d \sigma^{C}}{d \Omega}\right)} z_{M}^{2}\left(\left(x_{M}-x_{S}\right)^{2}+\left(y_{M}-y_{S}\right)^{2}+\left(z_{M}-z_{S}\right)^{2}\right) .
$$

In fact, this is only a first estimate of $n_{e}$. If the attenuation factor is taken into account, equation (51) would have the form:

$$
\begin{gathered}
n_{e}\left(x_{M}, y_{M}, z_{M}\right)=\widetilde{g}\left(x_{M}, y_{M}, \theta\right) \frac{4 \pi}{f_{0}\left(\frac{d \sigma^{C}}{d \Omega}\right)} \frac{1}{\mathcal{A}_{\mu}(S M, M D)} \\
z_{M}^{2}\left(\left(x_{M}-x_{S}\right)^{2}+\left(y_{M}-y_{S}\right)^{2}+\left(z_{M}-z_{S}\right)^{2}\right) .
\end{gathered}
$$

These considerations suggest the formulation of an attenuation correction algorithm as described in the following steps. Since $\mu$ is unknown, $\mathcal{A}_{\mu}(S M, M D)$ is also unknown. We aim to obtain the best possible estimate of $n_{e}$ using equation (46) with what is available at hand, i.e. equation (51).

Thus starting from actually collected data on the detector $\widetilde{g}(\mathbf{D}, \theta)$, identified as the zeroth step data $\widetilde{g}^{0}(\mathbf{D}, \theta)$, the use of equation (51) would yield a first estimate of the electronic density which shall be called $n_{e}(0)$. From now on to simplify notation we shall drop the spatial dependence of $n_{e}(\mathbf{r})$ as stated in equation (46) and denote the $j^{t h}$ step value of $n_{e}$ by $n_{e}(j)$. We obtain a first estimate of $\mu$ using equation (46), which shall be denoted $\mu^{0}$ and then compute the attenuation factor $\mathcal{A}_{\mu^{0}}$ to obtain the first corrected estimate as:

$$
\frac{\widetilde{g}^{0}(\mathbf{D}, \theta)}{\mathcal{A}_{\mu^{0}}}=\widetilde{g}^{1}(\mathbf{D}, \theta)
$$

where the attenuation effect has been removed in this first step. Thus the correction is aimed at obtaining collected data in the absence of attenuation.

We can repeat the procedure starting from $\widetilde{g}^{1}(\mathbf{D}, \theta)$, instead of $\widetilde{g}^{0}(\mathbf{D}, \theta)$, reconstruct $n_{e}(1)$ using equation (51), compute $\mu^{1}$ (using equation (46)) and $\mathcal{A}_{\mu^{1}}$ (using equation (45)) and end up with $\widetilde{g}^{2}(\mathbf{D}, \theta)$. The iterative process will be stopped whenever the mean relative quadratic error $(M R Q E)_{n_{e}}$ between two consecutive values 
$n_{e}(j-1)$ and $n_{e}(j)$, for $j=1,2, \ldots$, is less than a given threshold ( $1 \%$ for example). This process is called Iterative Attenuation Correction (IAC) algorithm and is summarized in figure 9.

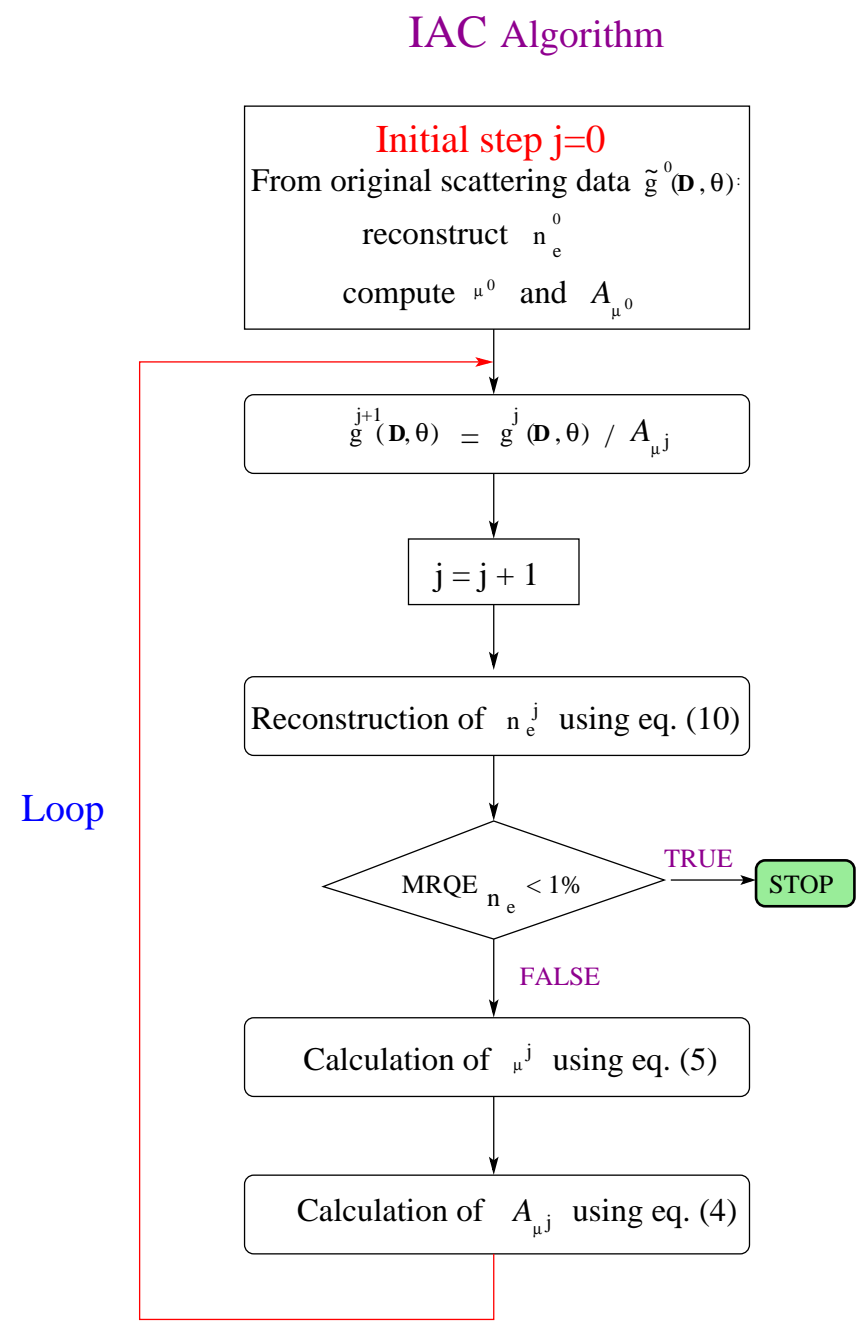

Figure 9. Flowchart of the Iterative Attenuation Correction (IAC) algorithm.

\subsection{Simulations Results}

Arguments showing the convergence of this iterative procedure are given in [16], as well as geometric limitations in real situations. To confirm the working we present simulations on medical phantoms under the following conditions.

We have chosen a phantom representing a thoracic cage in order to simulate features occurring in typical SPECT imaging studies, i.e. a non-homogenous object consisting of three different biological tissues (lungs, soft tissue and bone).

The object schematically representing a human thorax is made up of three types of materials: bone (white), soft tissue (gray) and lungs (black). A cross section with ribs and spinal column, lungs and tissue is presented in figure 10 . 

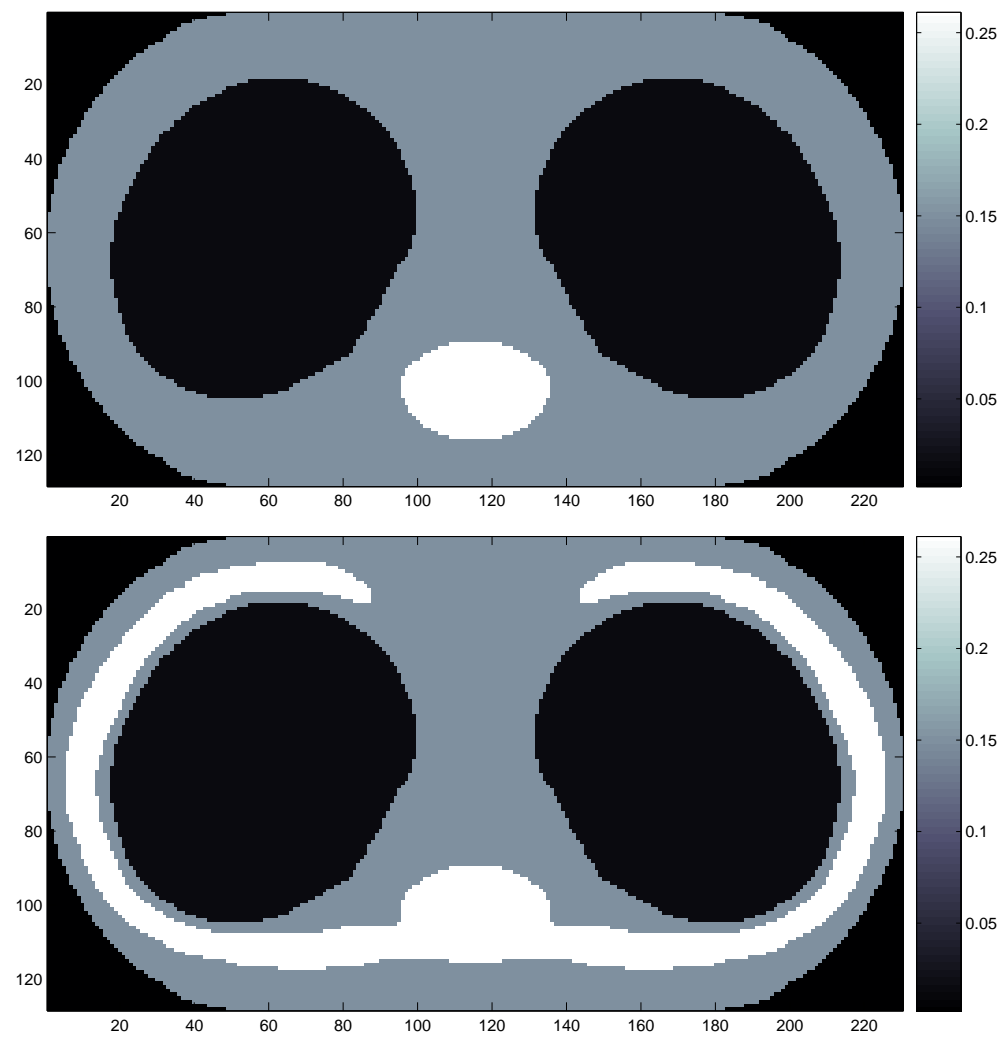

FiguRE 10. Representative slices of the original attenuation map of the object without ribs (top) and with ribs (bottom).

We adopt the following values of linear attenuation coefficients at $140 \mathrm{keV}$ photon energy: $0.28 \mathrm{~cm}^{-1}$ for bone, $0.15 \mathrm{~cm}^{-1}$ for water (soft tissues), $0.00017 \mathrm{~cm}^{-1}$ for air and $0.042 \mathrm{~cm}^{-1}$ for lungs. Typical values of electron density are: $5.91 \times 10^{23} \mathrm{~cm}^{-3}$ for bone, $3.34 \times 10^{23} \mathrm{~cm}^{-3}$ for water, $3.61 \times 10^{20} \mathrm{~cm}^{-3}$ for air and $0.634 \times 10^{23} \mathrm{~cm}^{-3}$ (inhale) or $1.632 \times 10^{23} \mathrm{~cm}^{-3}$ (exhale) for lungs.

In table 1, physical characteristic values for bone, water, air and lungs are given.

TABLE 1. Physical characteristics of some materials at $140 \mathrm{keV}$

\begin{tabular}{|l|l|c|c|}
\hline Material & $n_{e}\left(\mathrm{~cm}^{-3}\right)$ & Density $\left(\mathrm{g} . \mathrm{cm}^{-3}\right)$ & $\mu / \rho\left(\mathrm{cm}^{2} \cdot \mathrm{g}^{-1}\right)$ \\
\hline Bone & $5.91 .10^{23}$ & 1.85 & 0.143 \\
\hline Water & $3.34 .10^{23}$ & 1.00 & 0.150 \\
\hline Air & $3.61 .10^{20}$ & $1.210^{-3}$ & 0.139 \\
\hline Lung & see above & 0.3 & 0.139 \\
\hline
\end{tabular}

The gamma camera is placed above the voxel-based phantom at a distance of $80 \mathrm{~mm}$. The source, as shown in figure 8 , is placed at $20 \mathrm{~mm}$ nearby the volume under study on its side.

We present now results of the reconstruction of the medium electron density (and attenuation $\mu$ ) map from collected transmission data with the gamma camera operating in list mode. The detected photons are emitted 

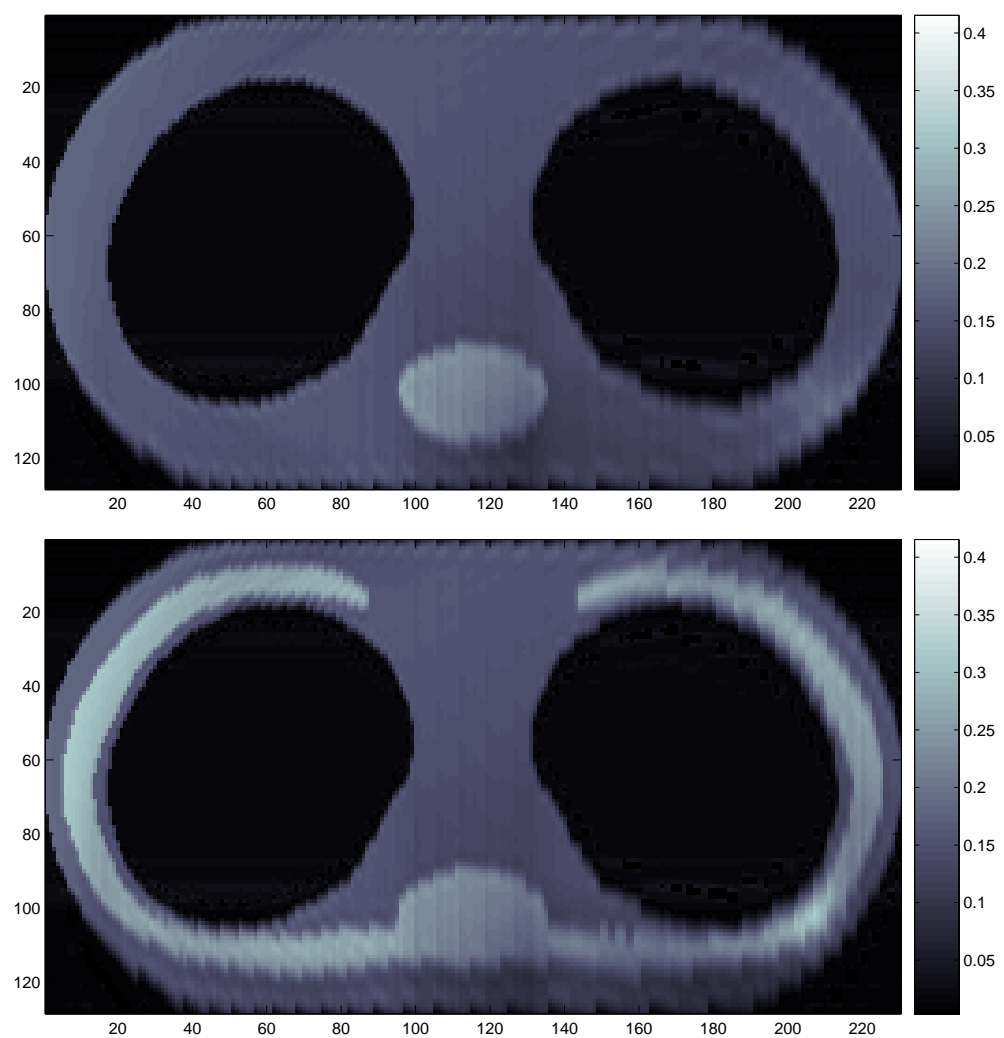

FiguRE 11. Representative slices of the reconstructed attenuation map corresponding to those shown in figure 10 .

by the external source and scattered (and attenuated) in the medium. We have used the reconstruction method presented and applied the IAC correction algorithm.

Figure 11 shows two representative slices of the 3D reconstructed transmission images. It can be seen that the left parts are more accurately recovered by the reconstruction procedure. This was expected since in our simulations, the transmission point source was on this side of the phantom (the gamma camera was placed above the medium, i.e. far from the spinal column).

The mean relative error $\left(M R E_{\mu}\right)$ between the original object, represented by $(\mu)$ and the reconstructed object represented by $\left(\mu^{*}\right)$, was used as a quantitative measure of image quality:

$$
M R E_{\mu}=\frac{1}{N} \sum_{\ell} \frac{\left|\mu^{*}(\ell)-\mu(\ell)\right|}{|\mu(\ell)|},
$$

where $N$ is the number of voxels of the voxel-based phantom, and $\ell$ the voxel label. It was demonstrated that a good performance can be achieved after only 8 iterations $\left(M R E_{\mu}=17.92 \%\right)$. The second figure of merit used is the mean relative quadratic error $\left(M R Q E_{\mu}\right)$ between the original and the reconstructed object, which is defined by

$$
M R Q E_{\mu}=\frac{1}{N} \sum_{\ell}\left(\frac{\mu^{*}(\ell)-\mu(\ell)}{\mu(\ell)}\right)^{2} .
$$

It was also shown that after 8 iterations, a good performance can be reached with $\left(M R Q E_{\mu}=16.82 \%\right)$. 


\section{Scattered Radiation in Transmission Imaging: Application to Non-Destructive-Testing}

In this section we discuss a new way of using scattered gamma radiation for non destructive material control. This has been called in the past Compton Tomography, see e.g. [17], and may be now called scatter transmission imaging as one collects not direct transmission radiation from an external source but scattered radiation emitted from such external source. Most devices invented so far make use of a fixed source emitting a pencil or a fan beam and a moving detector along a line (or equivalently a series of detector along this line).

In order to improve in sensitivity and resolution we have come up a new scanning device which is shown in Fig. 12 .

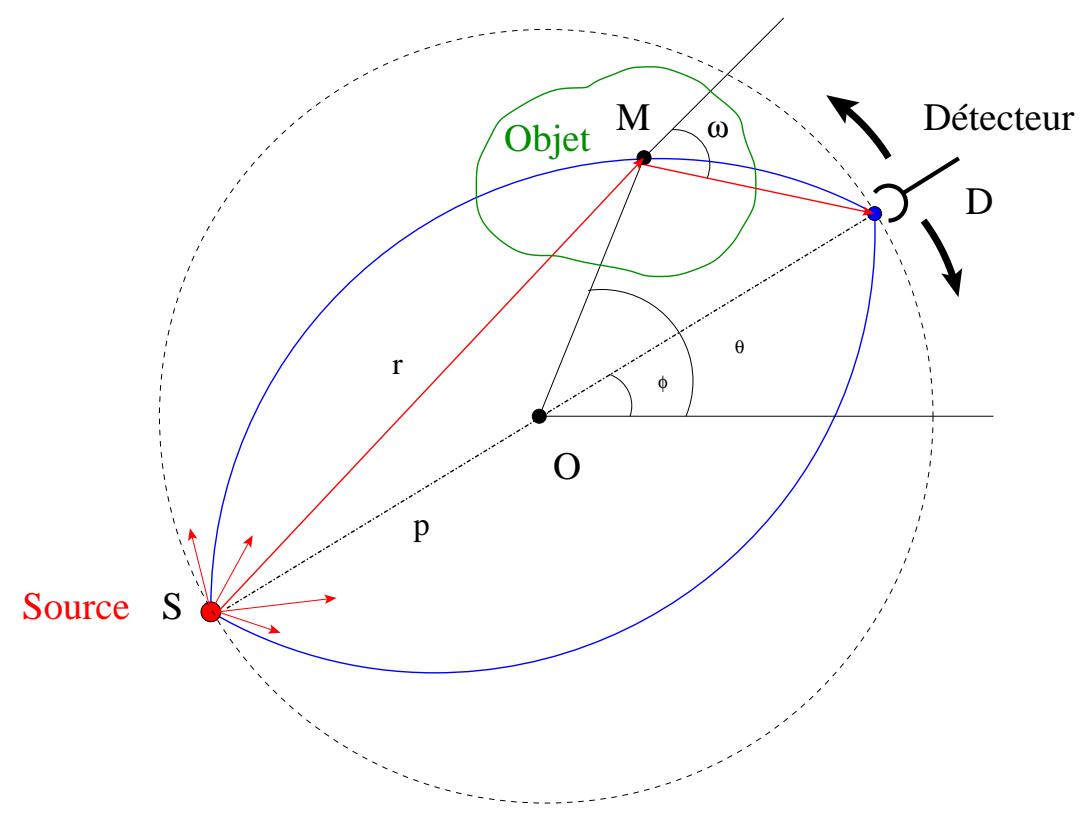

FigURE 12. New scattered gamma ray scanning for nondestructive evaluation

A source of gamma radiation is placed at $\mathbf{S}$ and allowed to emit in half-space facing the object. This incident gamma radiation will traverse an objet and scattered radiation is received at site $\mathbf{D}$, which is energy sensitive. The axis $S D$ can rotate around a center $\mathbf{O}$ and its position is labeled by an angle $\phi$. The distance $S D$ is fixed and is equal to $2 p$. It can be modified to fit the size of the scanned object between sites $\mathbf{S}$ and $\mathbf{D}$.

This is a tomographic device since it will explore a two dimensional section of an object. As it is clear from the mechanism of Compton scattering, the scattered photon intensity is proportional to the density of the scatterers in matter, namely the electrons. Thus by mapping this electronic density using the Compton effect, one is able to spot, detect and measure areas in bulk matter where this density undergoes drastic changes. This occurs when the scanning beam meets cracks, holes, inclusions or other types of structural defects. Hence such anomalies can be directly localized and evaluated.

So if $\mathbf{M}$ is a possible scattering site of the object, let $f(r, \theta)$ be the electronic density at $\mathbf{M}$, given by its polar coordinates $(r, \theta)$. The photon flux density received at $\mathbf{D}$ is called $\widehat{f}$ and is a function of $(\phi, \omega)$. Thus $\widehat{f}(\phi, \omega)$ is the image of $f(r, \theta)$ under this imaging map. As a function of two variables is mapped onto a function of two variables, the natural question to be raised is under which conditions $f(r, \theta)$ can be reconstructed from $\widehat{f}(\phi, \omega)$. The analysis of image formation by scattered rays will show that this mapping is a generalized Radon transform in two dimensions. 
For simplicity we assume that the source $\mathbf{S}$ emits isotropically $I$ photons per second in the $2 \pi$ radians around $\mathbf{S}$ (recall that we are in two dimensions). The photon flux density impinging on site $\mathbf{M}$ is thus

$$
\Phi_{0}=\frac{I}{2 \pi} \frac{1}{S M} .
$$

The fraction of photons scattered with an angle $\omega$ towards $\mathbf{D}$ is

$$
\frac{I}{2 \pi} \frac{1}{S M} f(\mathbf{M}) d V_{M} \sigma(\omega) d \Omega_{D}
$$

where $d \Omega_{D}=d a_{D} / M D$ is the angle under which site $\mathbf{D}$ is seen from $\mathbf{M}, f(\mathbf{M}) d V_{M}$ is the number of scatterers (electrons) around a two dimensional volume $d V_{M}$ at site $\mathbf{M}$. Hence the local photon flux density at $\mathbf{D}$ is

$$
\frac{I}{2 \pi} \frac{1}{S M} f(\mathbf{M}) d V_{M} \sigma(\omega) \frac{1}{M D}
$$

where $\sigma(\omega)$ is the Compton differential cross section $\sigma(\omega)=r_{e} P(\omega), r_{e}$, the classical radius of the electron, $P(\omega)$ the Klein-Nishina scattering probability

$$
P(\omega)=\frac{1}{2} \frac{1}{[1+\epsilon(1-\cos \omega)]^{2}}\left(1+\cos ^{2} \omega+\frac{\left(1-\cos ^{2} \omega\right)}{1+\epsilon(1-\cos \omega)}\right) .
$$

with $\epsilon=E_{0} / m c^{2}, m c^{2}$ being the rest energy of the electron and $E_{0}$ the initial energy of the gamma rays.

For fixed scattering angle $\omega$, the locus of scattering points $\mathbf{M}$ such that the scattered photons are detected at site $\mathbf{D}$ consists of two arcs of circle subtending an angle $(\pi-\omega)$. Hence the photon flux density received is

$$
\widehat{f}(\phi, \omega)=\int_{\operatorname{Arcs}} d \mu_{ \pm} \frac{I}{2 \pi} \frac{1}{S M} f(r, \theta) \sigma(\omega) \frac{1}{M D}
$$

where $d \mu_{ \pm}$is the arc of circle measures for the integration of $f(r, \theta)$.

The arcs of circle subtending the angle $(\pi-\omega)$ are given, in polar coordinates centered at $O$, by

$$
r=p\left(\sqrt{1+\tau^{2} \sin (\theta-\phi)} \pm \tau \sin (\theta-\phi)\right) \quad \text { with } \quad \tau=\cot \omega
$$

The distances $S M$ and $M D$ are

$$
S M=\sqrt{r^{2}+p^{2}+2 r p \cos (\theta-\phi)} \quad M D=\sqrt{r^{2}+p^{2}-2 r p \cos (\theta-\phi)} .
$$

The photon flux density received at the detecting site $\mathbf{D}$ is

$$
\begin{aligned}
\widehat{f}(\phi, \tau) & =\frac{I}{2 \pi} r_{e} P(\omega) \int_{\phi}^{\phi+\pi} d \mu_{-}(\theta) \frac{1}{S M} \frac{1}{M D} f\left(p\left(\sqrt{1+\tau^{2} \sin ^{2}(\theta-\phi)}-\tau \sin (\theta-\phi)\right), \theta\right) \\
& +\frac{I}{2 \pi} r_{e} P(\omega) \int_{\phi-\pi}^{\phi} d \mu_{+}(\theta) \frac{1}{S M} \frac{1}{M D} f\left(p\left(\sqrt{1+\tau^{2} \sin ^{2}(\theta-\phi)}+\tau \sin (\theta-\phi)\right), \theta\right),
\end{aligned}
$$

with the integration measures

$$
d \mu_{ \pm}(\theta)=\frac{p\left(\sqrt{1+\tau^{2} \sin (\theta-\phi)}-\tau \sin (\theta-\phi)\right)}{\sin \omega \sqrt{1+\tau^{2} \sin ^{2}(\theta-\phi)}} d \theta
$$

This is the form of the sought generalized Radon transform. Unfortunately no inversion formula is available for the moment. But it is worth while to see whether the idea is viable or not. In this spirit, we have performed 


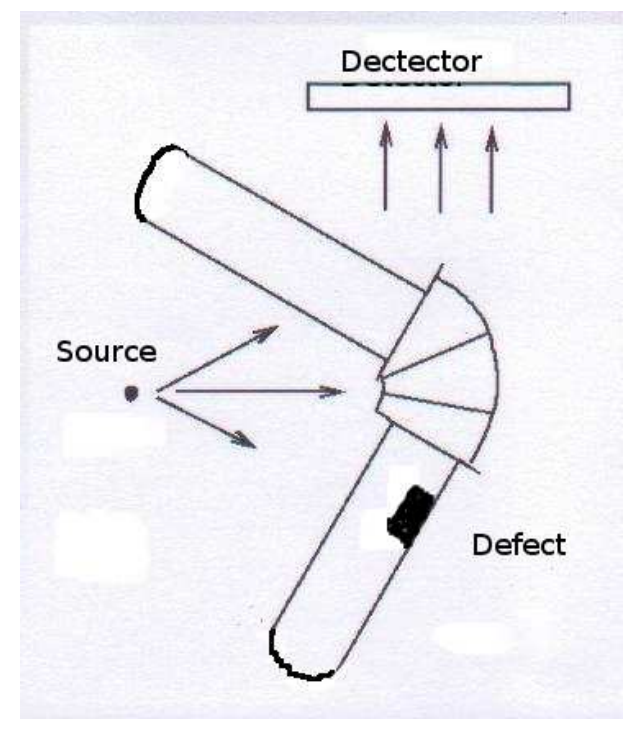

FIGURE 13. Setup for nondestructive testing of a pipe (3D view)

numerical object reconstruction based on this formula. Fig. 13 shows a right angle pipe which is taken as an object to be examined.

The next two figures (Fig. 14 and Fig. 15) show representations of this pipe without and with a built-in defect.

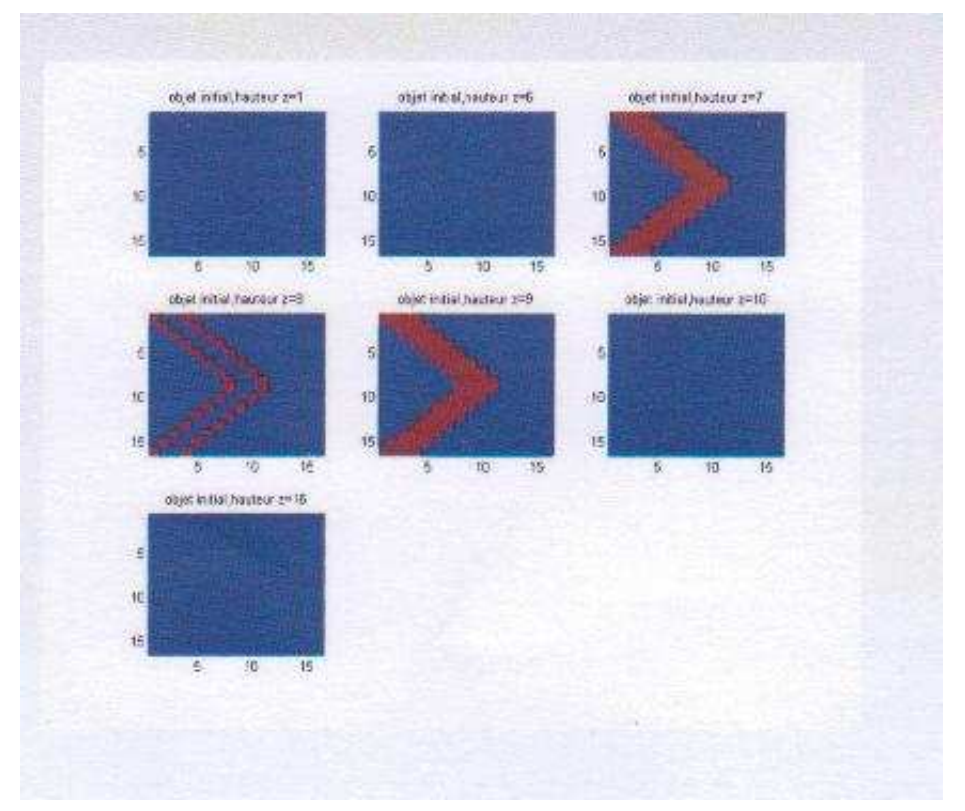

FiguRE 14. Original pipe without defect (plane by plane views)

Numerical inversion of the previous imaging equation yields the reconstructed pipe without and with defect, see Fig. 16 and Fig. 17. 


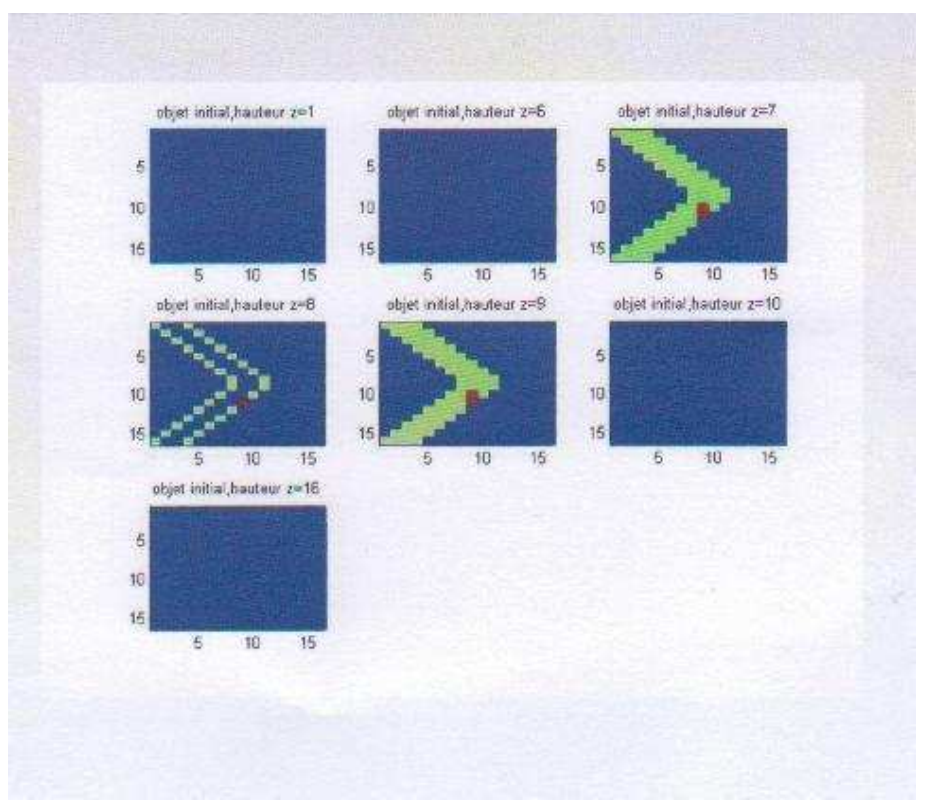

Figure 15. Original pipe with a defect (plane by plane views)
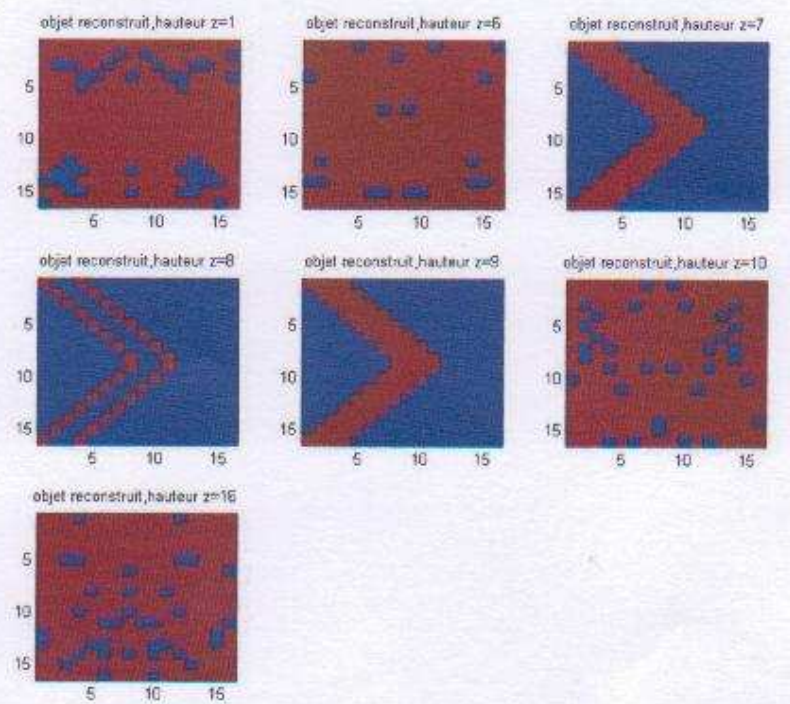

FiguRE 16. Reconstructed pipe without defect (plane by plane views)

With this preliminary experimental results, we are confident that the path to analytic inversion of the generalized Radon transform describing this scanning process with scattered radiation is worth while exploring. Note that this proposal is very different from the pencil beam scanning using double Compton scattering, which we have advocated before [22]. 


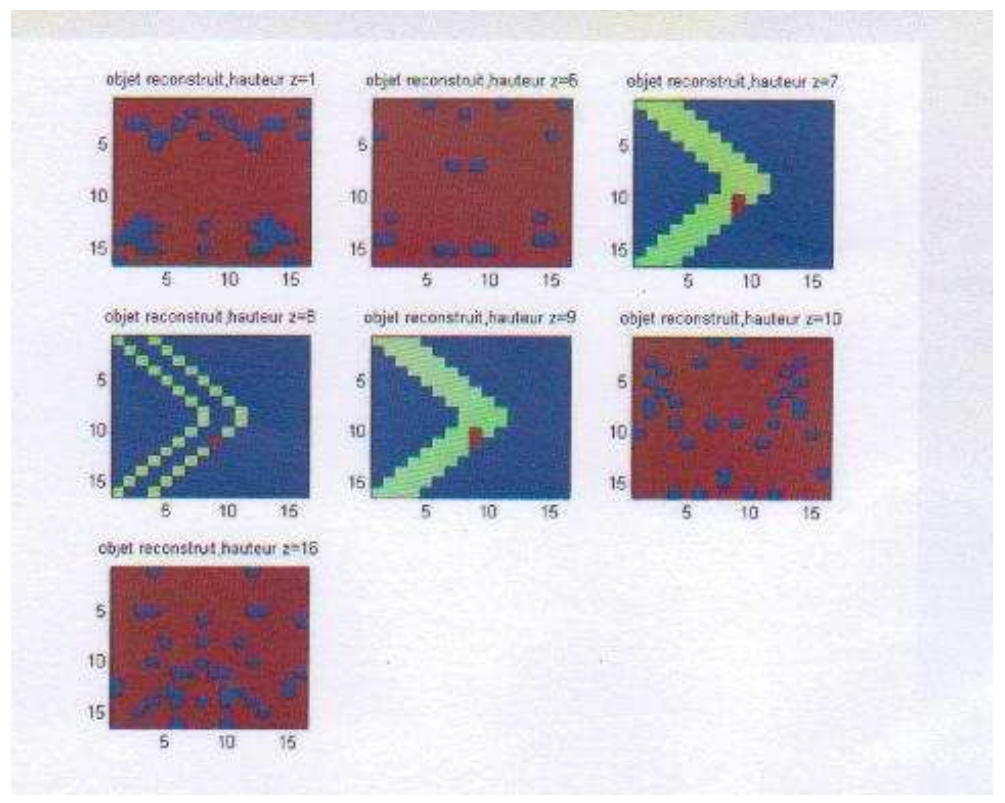

FigurE 17. Reconstructed pipe with a defect (plane by plane views)

\section{Conclusions and Perspectives}

In this work we have given theoretical results and numerical simulations showing the feasibility of object reconstruction using Compton-scattered gamma radiation in emission imaging. The new linear integral transform as well as its kernel (SPSF) are derived directly from a Compton-scattering physical analysis [13], which has been strongly recommended to be included in quantitative imaging by experts of the field [19]. The new imaging principle proposed here takes advantage of properties of scattered rays instead of rejecting them as usually done in most actual scatter correction methods. It would improve the signal-to-noise ratio, and consequently the contrast. Moreover, the fact that the angle of scattering is a free parameter is used here as a disposable parameter to collect data, the multi-views of the object are obtained without the need of moving the detector. This possibility is particularly interesting in the applications where the number of measurements is very limited.

Several perspectives can be opened from here. This work suggests a new scattered radiation-based emission/transmission imaging system having many advantageous properties: use of the same detector, same radionuclide, stationary acquisition for transmission scanning, etc. More importantly, what has been done for nuclear medicine imaging can be extended, in the same spirit, to other industrial applications such as non destructive testing. Another research direction is the development of a high sensitivity scatter radiation imaging principle which consists of operating the same principle but discarding the mechanical collimation. One can expect to gain drastically in sensitivity and field of view. This work is presently in progress. Finally on the theoretical level, a tantalizing challenge to be tackled is naturally the analytic inversion of the attenuated Compounded Conical Radon Transform (aCCRT) as a natural extension of the attenuated X-ray transform (for which a solution was found only recently by [18]. Such a solution would definitely confirm the imaging power of Compton scattered radiation proposed a few years ago [13]. 


\section{REFERENCES}

[1] H. H. Barrett and W. Swindell, Radiological Imaging I and II, Academic Press, New York, 1981.

[2] Berodias M G and Peix M G, "Nondestructive measurement of density and effective atomic number by photon scattering", Materials Evaluation 46 (8), pp. 1209-1213 (1998).

[3] Cesareo R, Borlino C C, Brunetti A, Golosio B and Castellano A, "A simple scanner for Compton tomography", Nuclear Instruments and methods in Physics Research A, 487, pp. 188-192 (2002).

[4] Clarke R L, Milne E N C and van Dyk G, "The use of Compton scattered gamma rays for tomography", Investigative Radiology 11 (May-June), pp. 225-235 (1976).

[5] M. J. Cree and P. J. Bones, "Towards direct reconstruction from a gamma camera based on Compton scattering", IEEE Trans. Medical Imaging, 13 (2), pp. 398-407 (1994).

[6] Davies B Integral Transforms and their Applications, Springer, New York, 1981.

[7] L. Eglin, Imagerie Scintigraphique: Modélisation et Restauration Multi-énergétiques, Ph.D Thesis, Université de CergyPontoise, 2002.

[8] Gerl J, "Gamma-ray imaging exploiting the Compton effect", Nuclear Physics A 752, pp. 688c-695c (2005)

[9] Harding G, "Inelastic photon scattering: effects and applications in biomedical science and industry", Radiat. Phys. Chem. 50 (1), pp. 91-111 (1997).

[10] Johns P C, Leclair R J and Wismayer M P, "Medical X-ray Imaging with scattered Photons", Opto-Canada: SPIE regional meeting on Optoelectronics, Photonics, and Imaging SPIE TD01, pp. 355-357 (2001).

[11] N. N. Lebedev, Special Functions and their Applications Dover, New York, 1955.

[12] Nguyen M K, Faye C, Eglin L and Truong T T, "Apparent Images Formation by Compton-Scattered Photons in Gamma-Ray Imaging", IEEE Signal Processing Letters 8(9), pp. 248-251 (2001).

[13] Mai K. Nguyen and T. T. Truong, "On an integral transform and its inverse in nuclear imaging", Inverse Problems, 18 (1), pp. 265-277 (2002).

[14] Mai K. Nguyen and T. T. Truong, "Exact inversion of a Compounded Conical Radon Transform and a novel nuclear imaging principle", C.R. Acad. Sci. Paris, Ser. I 335, pp. 213-217 (2002).

[15] Nguyen M K, Truong T T, Bui H D and Delarbre J L, "A novel inverse problem in $\gamma$-rays emission imaging", Inverse Problems in Science and in Engineering, 12 (2) pp. 225-246 (2004).

[16] Nguyen M K, Truong T T, Delarbre J L, Roux C, and Zaidi H, "Novel approach to stationary transmission scanning using Compton scattered radiation", Physics in Medicine and Biology, 52, pp. 4615-4632 (2007).

[17] S. J. Norton, "Compton scattering tomography", J. Appl. Phys., 76 (4), pp. 2007-2015 (1994).

[18] R. Novikov, "Une formule d'inversion pour la transformation d'un rayonnement X atténué", C. R. Acad. Sci.Paris, Ser. I 332 , pp. 1059-1063 (2001)

[19] M. S. Rosenthal, J. Cullom, W. Hawkins, S. C. Moore, B. M. W. Tsui and M. Yester, "Quantitative SPECT imaging : A review and recommendations by the focus committee of the society of nuclear medicine computer and instrumentation council," $J$. Nucl. Med., Vol. 36, pp. 1489-1513 (1995).

[20] M. Singh, "An electronically collimated gamma camera for single photon emission computed tomography", Med. Phys., 10, pp. 421-427 (1983).

[21] Todd R, Nightingale J and Everett D, ”A proposed gamma camera" Nature 251, pp. 132-134 (1974).

$[22]$ T. T. Truong, Mai K. Nguyen, H. D. Bui and C. Daveau, "Determination of the electronic density in a medium by an inverse method based on double-Compton scattering in transmission imaging", Proceedings of the 4th International Conference on Inverse Problems in Engineering: Theory and Practice, Rio de Janeiro, pp. 405-412, May 2002.

[23] H. Zaidi, "Relevance of accurate Monte Carlo modeling in nuclear medical imaging", Med. Phys. 26, pp. 574-608 (1999). 